Check for updates

Cite this: Phys. Chem. Chem. Phys., 2019, 21, 9810

Received 22nd October 2018 Accepted 18th April 2019

DOI: $10.1039 / c 8 c p 06573$

rsc.li/pccp

\title{
Improving the accuracy of Cu(II)-nitroxide RIDME in the presence of orientation correlation in water-soluble $\mathrm{Cu}(\mathrm{II})$-nitroxide rulers $\dagger$
}

\author{
Irina Ritsch, $\ddagger^{\mathrm{a}}$ Henrik Hintz, $\ddagger^{\mathrm{b}}$ Gunnar Jeschke, (iD ${ }^{\mathrm{a}}$ Adelheid Godt* ${ }^{\mathrm{b}}$ and \\ Maxim Yulikov (iD) *a
}

\begin{abstract}
Orientation selection is a challenge in distance determination with double electron electron resonance (DEER) spectroscopy of rigid molecules. The problem is reduced when applying the Relaxation-Induced Dipolar Modulation Enhancement (RIDME) experiment. Here we present an in-depth study on nitroxide-detected RIDME in $\mathrm{Cu}(I)$-nitroxide spin pairs using two $\mathrm{Cu}(\|)$-nitroxide rulers that are both water soluble and have comparable spin-spin distances. They differ in the type of the ligand (TAHA and PyMTA) for the Cu(II) ion which results in different contributions of exchange coupling. Both rulers feature substantial orientation correlation between the molecular frames of the $\mathrm{Cu}(\|)$ complex and the nitroxide. We discuss how the spin-spin couplings can be accurately measured and how they can be correlated to the nitroxide resonance frequencies. In that, we pay particular attention to the suppression of nuclear modulation and of echo crossing artefacts, to background correction, and to orientation averaging. With a nitroxide observer sequence based on chirp pulses, we achieve wideband detection of all nitroxide orientations. Two-dimensional Fourier transformation of data obtained in this manner affords observer-EPR correlated RIDME spectra that enable visual understanding of the orientation correlation. The syntheses of the $\mathrm{Cu}(\|)$-nitroxide rulers are presented. The synthetic route is considered to be of general use for the preparation of [metal ion complex]-nitroxide rulers, including water soluble ones.
\end{abstract}

\section{Introduction}

Electron Paramagnetic Resonance (EPR) spectroscopy is a powerful tool to study magnetic interactions in spin pairs. ${ }^{1-6}$ Currently, there are at least two main areas where interactions between nitroxide radicals and $\mathrm{Cu}$ (II)-based paramagnetic centres are of significant interest. Firstly, compounds containing strongly exchange-coupled $\mathrm{Cu}(\mathrm{II})$-nitroxide spin pairs have gained interest as potential switchable magnetic materials. ${ }^{7-16}$ In this context early work on accurate determination of the dipolar and exchange interactions in $\mathrm{Cu}(\mathrm{II})$-nitroxide ${ }^{17-22}$ and $\mathrm{Cu}(\mathrm{II})-\mathrm{Cu}$ (II) model compounds ${ }^{15,21,23,24}$ has gained new interest. In recent work it was demonstrated that such measurements reveal otherwise inaccessible information on the electronic structure of the

\footnotetext{
${ }^{a}$ Laboratory of Physical Chemistry, Department of Chemistry and Applied Bioscience, ETH Zurich, Vladimir-Prelogweg 2, 8093 Zurich, Switzerland. E-mail:maxim.yulikov@phys.chem.ethz.ch

${ }^{b}$ Faculty of Chemistry and Center for Molecular Materials $\left(\mathrm{CM}_{2}\right)$, Bielefeld University, Universitätsstraße 25, 33615 Bielefeld, Germany. E-mail: godt@uni-bielefeld.de

$\dagger$ Electronic supplementary information (ESI) available: SI1: Supporting information for spectroscopic results; SI2: Supporting information for synthesis. See DOI: 10.1039/c8cp06573j

\# Authors contributed equally. I. R. performed the spectroscopic measurements and data processing. H. H. performed the synthetic work.
}

systems. ${ }^{16,25}$ Secondly, complexes of $\mathrm{Cu}(\mathrm{II})$ ions are attracting attention in bio-EPR spectroscopy, ${ }^{26-29}$ both as native metal centres in proteins, ${ }^{23,26,30-38}$ and as spin labels for orthogonal pulse dipolar spectroscopy. ${ }^{39,40}$ The potential of the latter approach has been recognised previously and is reflected in a growing interest in $\mathrm{Cu}$ (II)-based spin labelling strategies..$^{17,26,29,40,41}$ Preparation of orthogonally spin labelled samples often requires some additional experimental effort, which is why orthogonal spin labelling methods may continue to play a minor role in structure determination of monomeric biomolecules. Their strength, however, lies in the possibility of spectroscopic selection in systems of several interacting molecules, and they thus may well become a method of choice in the studies of intermolecular biomolecule interactions. ${ }^{40-49}$

Both mentioned fields of application require an appropriate choice of spectroscopic method, which depends on the strength of the spin-spin interaction of interest. Interactions that are typically relevant in EPR spectroscopy can differ by orders of magnitude, depending on the coupled spins. While magnetic interactions of the strength of hundreds or thousands of $\mathrm{MHz}$ are directly visible in the continuous-wave (CW) EPR line shapes, the detection of moderate (ten to hundred $\mathrm{MHz}$ ) and weak (few $\mathrm{MHz}$ or weaker) spin-spin interactions requires use of dedicated pulse EPR techniques, generally referred to as pulse dipolar EPR spectroscopy (PDS). The most commonly applied pulse experiments are 
Double Quantum Coherence (DQC), ${ }^{52}$ Single Frequency Technique for Refocusing (SIFTER), ${ }^{53-55}$ Double Electron Electron Resonance (DEER, also known as PELDOR), ${ }^{3,56}$ the ' $2+1$ ' pulse sequence, ${ }^{57}$ and Relaxation Induced Dipolar Modulation Enhancement (RIDME) $^{58,59}$ experiment. Our labs currently focus on biological applications of dipolar spectroscopy, which in most cases implies the weak coupling regime. The most commonly used experiment in this field is the DEER technique, which has been very well studied and optimized for detecting nitroxide-nitroxide interactions. PDS experiments on $\mathrm{Cu}(\mathrm{II})$-nitroxide pairs pose additional challenges. Sensitivity of PDS experiments on $\mathrm{Cu}$ (II) centres suffers from the small fraction of spins within the excitation band of a typical rectangular microwave pulse. This limits the dipolar modulation depth, when pumping $\mathrm{Cu}$ (II) spins, or the echo signal-to-noise ratio (SNR), when detecting them. The fraction of excited $\mathrm{Cu}$ (II) centres generally decreases with increasing spectrometer frequency, because the spectral width of $\mathrm{Cu}$ (II) depends on the $g$-tensor anisotropy, which is the dominating factor for spectral width already at X-band frequencies around $9 \mathrm{GHz}$. The appearance of the Cu(II) EPR spectrum varies strongly with the type of ligand. The $g$-value ranges and the corresponding resonance field ranges for $\mathrm{Cu}$ (II) complexes and nitroxide radicals usually do not overlap, but some cases of their overlap at the low- $g$ range for $\mathrm{Cu}$ (II) can be found. However, due to other anisotropic terms, most importantly the $\mathrm{Cu}$ (II) hyperfine interaction, it is possible that the EPR spectra of $\mathrm{Cu}$ (II) and nitroxide overlap at lower frequencies and do not overlap above some threshold frequency/field. Here we address such a case, when at our detection frequency of $35 \mathrm{GHz} \mathrm{Cu}$ (II) and nitroxide EPR spectra do not overlap.

In DEER spectroscopy the fraction of spins that contribute to the experiment can be increased by applying frequency swept pulses. ${ }^{60-64}$ Excitation bandwidths of the order of $1 \mathrm{GHz}$ can be routinely achieved with modern arbitrary waveform generators (AWGs) and with specially constructed wideband pulse EPR setups $^{65}$ and resonators. ${ }^{66}$ This performance is fully adequate for $\mathrm{Cu}(\mathrm{II})$-nitroxide DEER at X-band frequencies (9.5 GHz). At Q band frequencies (34-36 GHz), where concentration sensitivity is higher, and at yet higher frequencies, where absolute sensitivity is higher, bandwidth limitations are experienced for $\mathrm{Cu}(\mathrm{II})$ even with the best available spectrometers.

Another complication in $\mathrm{Cu}(\mathrm{II})$-nitroxide PDS measurements arises from the strong correlation between the resonance field and the orientation of the molecular coordinate frame with respect to the external magnetic field for both spin types. ${ }^{67}$ On the one hand, such orientation selection can provide additional information of the geometry of the spin pair. Such approaches have been developed for the case of nitroxides and other organic radicals, ${ }^{68-70}$ which have led to several application studies. $^{71-75}$ On the other hand, orientation selection significantly complicates experiments and data analysis if only the distance distribution is of interest. In order to obtain the full information, PDS data need to be measured over a broad range of detection and pump positions, which is tedious for the broad $\mathrm{Cu}(\mathrm{II})$ EPR spectra. The full information may even be inaccessible if spectral width strongly exceeds resonator bandwidth.
Instead of using a pump pulse for flipping the spins B coupled to observer spins A, as is done in DEER spectroscopy, a longitudinal-relaxation mixing block $(\pi / 2)-T-(\pi / 2)$ can be used that has no bandwidth limitation. This is the basis of the relaxation-induced dipolar modulation enhancement (RIDME) experiment. ${ }^{58,59}$ The performance of this experiment correlates with the ratio of the longitudinal relaxation time of the B spins to the transverse relaxation time of the A spins. Relaxation behaviour dictates that in the $\mathrm{Cu}$ (II)-nitroxide RIDME experiment, the nitroxide spin takes the role of the observer spins A (slow longitudinal relaxation) and the $\mathrm{Cu}(\mathrm{II})$ centres the role of the flipped spins B (fast longitudinal relaxation). In this configuration high-quality $\mathrm{Cu}(\mathrm{II})$-nitroxide RIDME data can be obtained. ${ }^{28,29}$ It also results in an essentially infinite excitation bandwidth for the $\mathrm{B}$ spins, while the detection sequence for the nitroxide radicals can be broadbanded or selective, depending on the chosen microwave pulse parameters. Importantly, since RIDME is a single-frequency experiment, there are no limitations in placing the detection frequency within the nitroxide spectrum, or even exciting the entire spectrum. These considerations suggest that it should be feasible to perform practically complete orientation averaging in RIDME experiments, a feat that is very difficult to accomplish with $\mathrm{Cu}$ (II)-nitroxide DEER. For a nitroxide-[Cu(II)(terpy) $\left.{ }_{2}\right]$-nitroxide model compound a five point averaging of the RIDME traces over the nitroxide spectrum led to dipolar frequency patterns reasonably close to the expected Pake pattern with somewhat suppressed parallel components. ${ }^{28}$ In contrast to high-spin metal centres, where dipolar frequency overtones in RIDME data complicate distance analysis, ${ }^{76}$ the analysis of $\mathrm{Cu}(\mathrm{II})$-nitroxide RIDME time-domain data is practically the same as that for DEER experiments on nitroxide pairs.

Previous $\mathrm{Cu}(\mathrm{II})$-nitroxide RIDME work focused on determination of the relative orientations of paramagnetic centres, ${ }^{28}$ and on characterization of metal-chelator complexation equilibria. ${ }^{29}$ Here, we address the issue of accurate determination of the dipolar frequency distribution, which is essential for obtaining an artefactfree distance distribution. In addition to orientation averaging, this requires a more detailed analysis of the different types of artefacts in RIDME data. We have recently discussed such artefacts for Gd(III)-Gd(III) RIDME. ${ }^{77}$ In the course of the present study, we discovered a deviation of the intermolecular RIDME decay from the stretched exponential decay function commonly used for background correction. This deviation, which had not previously been noticed, occurs for the commonly used timing of the experiment and can distort $\mathrm{Cu}$ (II)-nitroxide distance distributions.

For the spectroscopic experiments geometrically well-defined model compounds were needed. Furthermore, to be able to perform the experiments under conditions used to study biomolecules, solubility in water is requested. Therefore, as a part of the study we had to develop a synthetic route providing access to two water soluble $\mathrm{Cu}(\mathrm{II})$-nitroxide rulers and variations thereof in respect to spin-spin distance, type of ligand and type of metal ion. The RIDME experiments on such water-soluble rulers demonstrated that spectroscopic properties of $\mathrm{Cu}$ (II)-nitroxide spin pairs in water/ glycerol mixtures are very similar to the corresponding properties determined in earlier works in organic solvents, which allows to 


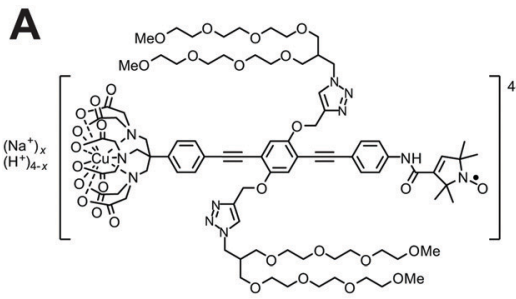

[Cu(II)-TAHA]-nitroxide ruler 1

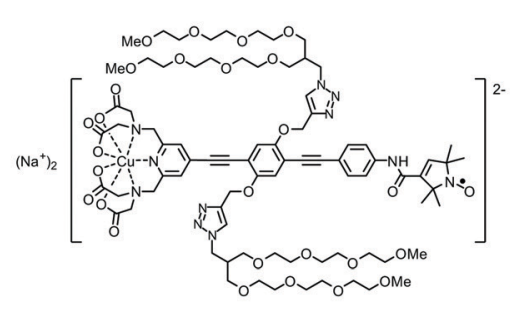

[Cu(II)-PyMTA]-nitroxide ruler 2

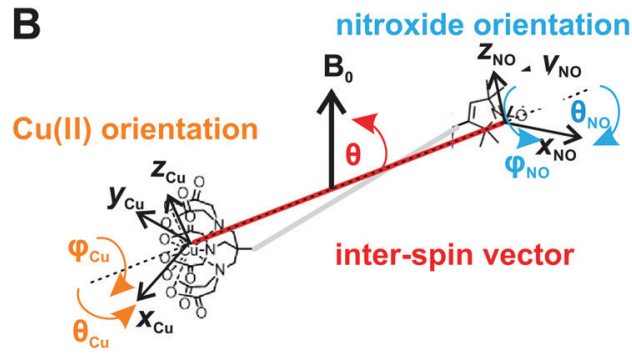

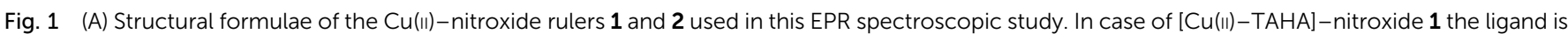

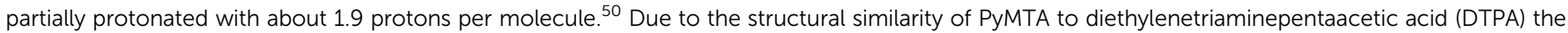

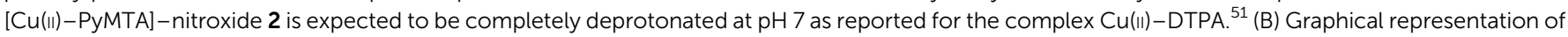
the relevant angles for orientation selection.

use the entire information obtained so far in different $\mathrm{Cu}(\mathrm{II})-$ nitroxide RIDME studies also for planning biological applications.

This paper is organised as follows: first, the details on the spectroscopic methods are given. Next, the syntheses of the herein used $\mathrm{Cu}(\mathrm{II})$-nitroxide rulers 1 and 2, [Cu(II)-TAHA]-nitroxide and [Cu(II)-PyMTA]-nitroxide, (Fig. 1(A)) are described.

We then discuss ESEEM artefacts in our RIDME measurements. Furthermore, we report a previously unnoticed background distortion and show that it can be avoided by increasing the first interpulse delay at the expense of some modest sensitivity reduction. We next discuss echo crossing artifacts. Then the performance of the $\mathrm{Cu}(\mathrm{II})$-nitroxide RIDME technique when applied to [Cu(II)-TAHA]-nitroxide is discussed and compared to the published data of nitroxide-[Cu(II)(terpy) 2$]$-nitroxide.$^{28,29}$ Here we show an optimisation of experimental parameters concerning measurement temperature and mixing time. We continue with demonstrating virtually complete orientation averaging by summation of the RIDME traces measured at different observer fields, and alternatively by wideband RIDME measurements with AWG-generated chirp pulses. The latter experiment affords twodimensional nitroxide-EPR correlated dipolar spectra, which contain information on orientation correlation between the spin-spin vector and the nitroxide molecular frame. We then report on the performance of the optimised experimental conditions on a second $\mathrm{Cu}(\mathrm{II})$-nitroxide ruler with $\mathrm{Cu}$ (II)-PyMTA as the spin label. Finally, we compare the RIDME experiment to an ultrawideband (UWB) DEER measurement at Q-band.

\section{Spectroscopic methods}

\section{Sample preparation and RIDME setup with rectangular pulses}

All RIDME experiments were performed at Q-band (34-36 GHz). The EPR samples were prepared as $100 \mu \mathrm{M}$ or $200 \mu \mathrm{M}$ solutions in a mixture of $\mathrm{D}_{2} \mathrm{O}$ and $\mathrm{d}_{8}$-glycerol in a $1: 1$ volume ratio. The measurement temperature was varied between $10 \mathrm{~K}$ and $50 \mathrm{~K}$, using an Oxford Instruments He-flow cryostat system. For each sample, the $\mathrm{Cu}(\mathrm{II})$-nitroxide ruler solution was filled into a $3 \mathrm{~mm}$ outer diameter thin-wall quartz tube and shock frozen by quick immersion into liquid nitrogen.

For the RIDME setup with rectangular microwave (m.w.) pulses, we used a home-built resonator, accommodating large-diameter samples, ${ }^{79}$ and a commercial Bruker E580 X/Q spectrometer upgraded to a high m.w. power. ${ }^{80}$ The conventional rectangularpulse setup is shown in Fig. 2(A). Unless stated otherwise the first inter-pulse delay $t_{1}$ was set to $4100 \mathrm{~ns}$ (see below for the reason), and the length of the second delay $t_{2}$ was set to $3500 \mathrm{~ns}$. The mixing time values were varied between different RIDME measurements. This is indicated in the text and in the figure captions. The lengths of the $\pi / 2$ pulses were set to either $12 \mathrm{~ns}$ ('non-selective rectangular pulses' setup), or to $50 \mathrm{~ns}$ ('selective rectangular pulses' setup). Accordingly, the lengths of the $\pi$ pulses were set either to $24 \mathrm{~ns}$ (non-selective), or to $100 \mathrm{~ns}$ (selective).

The stationary refocused virtual echo $^{59}$ that forms after the last $\pi$ pulse (in the following referred to as 'RIDME RVE echo') was detected with a detection window of twice the pulse length of the $\pi$-pulse. The eight-step phase cycle to remove crossing $e^{e} \cos ^{59}$ was used. An eight-step averaging cycle with a timeincrement of $16 \mathrm{~ns}$ and simultaneous $t_{1}$ and $t_{2}$ averaging was used to suppress unwanted electron-nuclear contributions to the RIDME time-evolution signal due to the matrix ${ }^{2} \mathrm{H}$ nuclei. ${ }^{77}$ More details on the electron-nuclear modulation averaging is given in the Results section.

\section{DEER measurements}

The comparative DEER measurement on [Cu(II)-TAHA]-nitroxide 1 was performed on the commercial Bruker spectrometer described above with an arbitrary waveform generator (AWG) (Agilent M8190A) setup plugged into the pump pulse channel. The experiment required a careful adjustment of the resonator mode (same resonator for $3 \mathrm{~mm}$ outer diameter samples as for the other experiments ${ }^{79}$ ) to yield a very broad, strongly overcoupled resonator mode. This reduced sensitivity at the detection frequency, but was necessary in our attempt to obtain significant spin inversion at the pump frequency, given the large frequency offset of almost $1 \mathrm{GHz}$. Such an adjustment of the resonator mode also distributed the total microwave power over a larger frequency range, resulting in smaller power spectral density/ smaller $B_{1}$ field at any frequency within the resonator profile. As a consequence, only relatively low critical adiabaticity could be achieved for the given durations of the chirp pulses, which was not sufficient to obtain full inversion of the nitroxide spectrum by the pump pulse. The DEER detection was performed at $30 \mathrm{~K}$ on the maximum of the $\mathrm{Cu}(\mathrm{II})$ spectrum with pulse lengths of $48 \mathrm{~ns}$, resp. $96 \mathrm{~ns}$ pulses (for $\pi / 2$, resp. $\pi$ nominal flip angle). The broad 

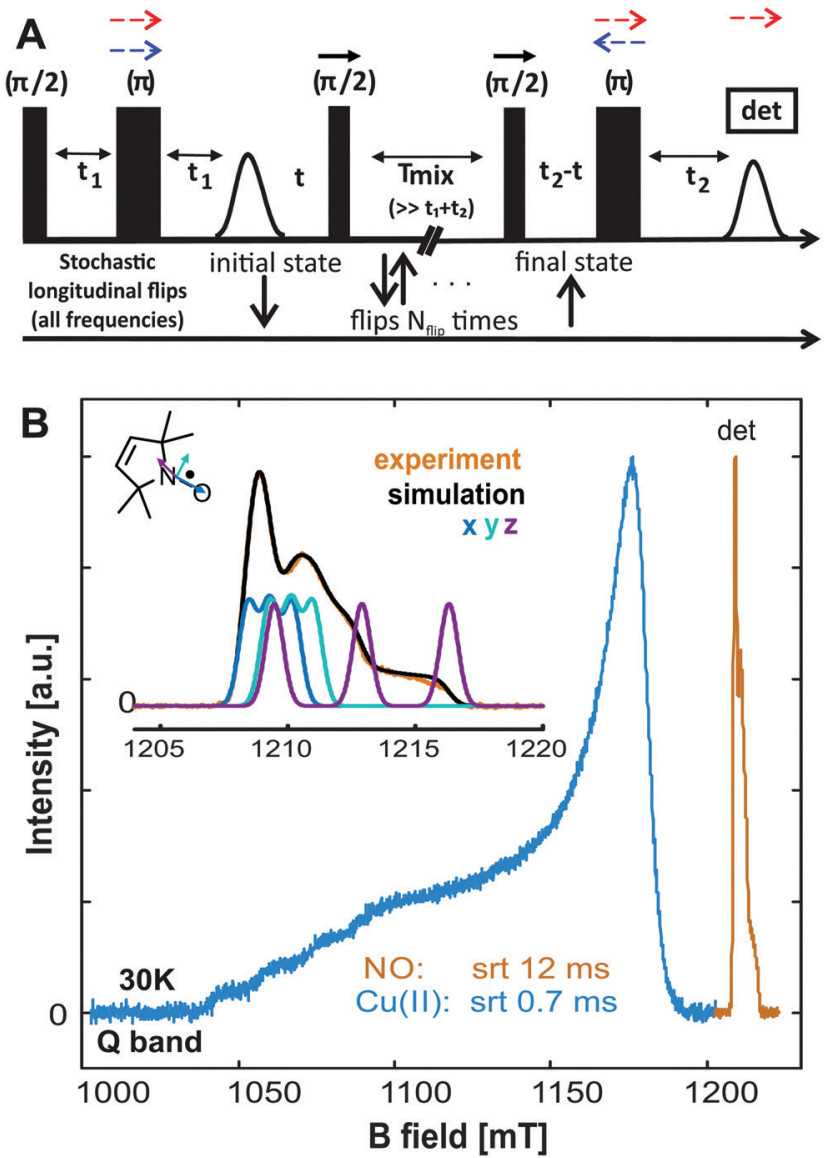

Fig. 2 (A) The 5-pulse RIDME sequence with first $\left(t_{1}\right)$, and second $\left(t_{2}\right)$ refocusing periods. Inversion of the dipolar coupled $\mathrm{Cu}(I)$ spins happens due to stochastic longitudinal flips during $T_{\text {mix }}$. The pulses that form the mixing block are stepped together (black arrows) to give the RIDME time axis $t$. The blue and red dashed arrows above the refocusing pulses indicate which pulses are moved in the ESEEM averaging schemes (see Section 3.2). (B) Q-band spectra of the $\mathrm{Cu}(\mathrm{II})$ (blue) and the nitroxide (orange) regions for ruler 1 at $30 \mathrm{~K}$. The maxima of the spectra are separated by approximately $33 \mathrm{mT}$. (inset) Zoom on the nitroxide spectrum and EasySpin ${ }^{78}$ fit (for parameters see SI1, ESI†). For maximum sensitivity, RIDME was performed on the maximum of the nitroxide spectrum, which may result in moderate orientation selection, as is illustrated by three simulated sub-spectra of the nitroxide powder spectrum (EasySpin pepper function), which correspond to orientations of the magnetic field along the principle axes of the nitroxide $g$-tensor.

resonator mode used in this setup did not allow for harder pulses due to insufficient microwave power. The delays were set to $t_{1}=400 \mathrm{~ns}$, and $t_{2}=3500 \mathrm{~ns}$. In order to maximise modulation depth we aimed at performing an inversion pulse over the full range of the nitroxide spectrum. To this end we used a linear up-chirp pump pulse with a bandwidth of $450 \mathrm{MHz}$, and an offset from the detection frequency of $+900 \mathrm{MHz}$, with a pulse duration of $150 \mathrm{~ns}$.

\section{RIDME setup with coherent chirped broad-band pulses}

The RIDME experiments with chirped pulses were performed on the home-built coherent AWG spectrometer ${ }^{63}$ and with the same type of resonator ${ }^{79}$ for oversized samples ( $3 \mathrm{~mm}$ tubes) as was used in the rectangular pulses setup. The resonator profile and pulse excitation bandwidth characterisation are shown in
Fig. S23 (ESI $\dagger$ ). To achieve refocusing of the resonance offsets at the detection position, a pulse length ratio of $2: 2: 2: 2: 1$ was used with all chirp pulses as up-sweeps. This setup allowed compensating for different excitation time points of the nitroxide radicals with different resonance offsets as well as for compensating the dynamic Bloch-Siegert phase shifts from the primary $\pi / 2$ pulse and the two refocusing $\pi$ pulses. ${ }^{81}$ The Bloch-Siegert dynamic phase shifts for the two $\pi / 2$ pulses of the mixing block are not compensated in this scheme. This can be understood if we consider the mixing block as an extended $\pi$ pulse. However, since the dynamic phase shift is approximately proportional to the value of critical adiabaticity $\left(Q_{\mathrm{cr}}\right)$, the corresponding phase shift for the mixing block $\pi / 2$ pulses $\left(Q_{\mathrm{cr}}=2 \log 2 / \pi\right)$ is about an order of magnitude weaker than for the inversion $\pi$ pulses $\left(Q_{\mathrm{cr}}=5\right)$. The pulse lengths of the $\pi / 2$-pulses, resp. the long $\pi$-pulses were $t_{\mathrm{p}}=150 \mathrm{~ns}$, while the short $\pi$-pulses were set to $t_{\mathrm{p}}=75 \mathrm{~ns}$. All pulses were used with a half-sine rise period with $t_{\text {rise }}=15 \mathrm{~ns}$ to suppress ripples, ${ }^{63,82}$ and had a bandwidth of $400 \mathrm{MHz}$ for the measurement performed on ruler 1 , resp. $450 \mathrm{MHz}$ for the measurement performed on ruler 2 . Note that both pulse bandwidths exceed the spectral width of nitroxide at Q-band ( $\sim 250 \mathrm{MHz})$, but the exact choice was made to give the best result in each spectrometer session, due to the slightly different resonator profiles. The central frequency of the chirp pulse was placed at the centre of the nitroxide spectrum, and approximately at the centre of the resonator profile. More details for the setting up of the chirp pulses is given in the SI1 (ESI $\dagger$ ). For convenience, the same pulse settings were used for the mixing block $\pi / 2$ pulses and for the first $\pi / 2$-pulse. The same 8 -step phase cycle and ESEEM averaging cycle $(8 \times 16 \mathrm{~ns})$ as for the conventional RIDME experiments were used.

All chirp-RIDME experiments were conducted at $20 \mathrm{~K}$ with a mixing time of $T_{\text {mix }}=1.6 \mathrm{~ms}$ and at the condition $t_{1}=3.5 \mu \mathrm{s}$. The same stationary refocused virtual echo as in the experiments with rectangular pulses was detected (RIDME RVE echo). For orientation averaging the echo was integrated and the resulting dipolar evolution data were treated like a conventional RIDME trace. In addition, EPR-correlated dipolar spectra were computed by 2D Fourier transformation (FT) as follows. First, the transient time trace of the RIDME RVE echo for each position of the RIDME mixing block was apodized by a symmetric Chebychev window. FT of the RIDME RVE echo yielded the nitroxide EPR spectrum dimension. Then, the RIDME time trace at each discrete point in the nitroxide spectrum was fitted with a stretched exponential background function, the form factor was obtained by division of the primary data by the fit, and the constant offset was subtracted. After a second FT along the RIDME time dimension, i.e. the time that defines the starting position of the mixing block in the RIDME sequence, we obtained the EPR-correlated dipolar spectrum.

\section{Results and discussion}

\subsection{Synthesis of the $\mathrm{Cu}(\mathrm{II})$-nitroxide rulers}

The synthesis design was led by the goal of establishing a generally applicable procedure for the preparation of metal 
ion-nitroxide rulers which are soluble in water and water containing organic solvents. Such a solubility profile allows to test the spectroscopic tool under conditions used for its application to biomolecules. A further design criterion was that the ligand, which is needed for anchoring the metal ion, forms stable complexes with several metal ions of importance for metal ion-nitroxide EPR spectroscopy. ${ }^{17-22,43,46,49,84-91}$ To reach these goals the rod-like spacer was furnished with highly hydrophilic, branched oligo(ethyleneglycol) (PEG) chains and the ligands PyMTA ${ }^{66,92,93}$ and TAHA $^{83,92,94}$ were selected (Fig. 1). These ligands form readily $1: 1$ complexes with $\mathrm{Cu}$ (II) ions as well as with other paramagnetic ions, such as $\mathrm{Mn}$ (II) ${ }^{92}$ and $\mathrm{Gd}(\mathrm{III})^{94}$ and are of small size which becomes relevant when being used as spin labels. Cu(II)-nitroxide rulers and, more generally, compounds labeled with $\mathrm{Cu}$ (II) and nitroxide, reported in the literature, contain terpyridine (terpy), ${ }^{17,28,29}$ porphyrine, ${ }^{18}$ peptide-based binding loops, ${ }^{95-97}$ as the $\mathrm{Cu}$ (II) binding unit. The rulers with terpyridine were also used in combination with $\mathrm{Co}(\mathrm{II}), \mathrm{Mn}$ (II) and Gd(III) as the metal ions and were used already in the context of $\mathrm{Co}$ (II)-nitroxide and $\mathrm{Mn}$ (II)nitroxide RIDME. ${ }^{49,84,88}$ In contrast to the chosen ligands PyMTA and TAHA, terpyridine forms a $2: 1$ complex of the form nitroxide- $\left[\mathrm{Cu}(\mathrm{II})(\text { terpy })_{2}\right]$-nitroxide. Importantly, essentially all so far reported $\mathrm{Cu}(\mathrm{II})$-nitroxide RIDME experiments were performed on nitroxide- $\left[\mathrm{Cu}(\mathrm{II})(\text { terpy })_{2}\right]$-nitroxide, which is not water soluble. ${ }^{17,28,29}$

To obtain $\mathrm{Cu}(\mathrm{II})$-nitroxide rulers one has to connect a nitroxide, a ligand or a ready-made complex, and a spacer. As shown in Fig. 3, we attached the nitroxide to the spacer first, followed by the ligand. The reverse order should work as well, however, the chosen sequence is more efficient when different ligands are to be investigated. The PEG side chains were introduced after the complete scaffold had been assembled. This way, up to a rather late stage in the overall syntheses the compounds were simply isolated by standard extraction and standard column chromatography on silica gel. The spacer backbone was chosen to consist of alternating phenylene (P) and ethynylene (E) units because such $(\mathrm{PE})_{n}$ spacers provide sufficient shape persistence. ${ }^{98}$ Their number $n$ of repeating units and therefore their length is easily adjustable to meet a required distance between metal ion and nitroxide, and they are well accessible. ${ }^{99}$ In this work the syntheses of the rulers [Cu(II)-TAHA]-nitroxide $\mathbf{1}$ and [Cu(II)-PyMTA]-nitroxide 2 with rather short metal ion-nitroxide distances are presented. We expect the route to be applicable for the synthesis of longer metal ion-nitroxide rulers, as well. Indeed, [Cu(II)-PyMTA]-nitroxide with a (PE) ${ }_{4}$ spacer has been obtained following the same strategy (results will be published separately).

The syntheses of the rulers [Cu(II)-TAHA]-nitroxide $\mathbf{1}$ and [Cu(II)-PyMTA]-nitroxide 2 started from the phenyleneethynylene $(\mathrm{PE})_{1}$ spacer 3 (for details see SI2, ESI $\dagger$ ). ${ }^{99}$ To enable the attachment of the nitroxide it was equipped with an amino group through Sonogashira-Hagihara coupling with $p$-iodoaniline. To ease the separation of the target compound from the accompanying oxidative alkyne dimerization product the polar tagging strategy ${ }^{98-100}$ was applied. Reaction of the amino functionalised spacer $\mathbf{4}$ with the $\mathrm{N}$-acylpyridinium salt $\mathbf{6}$, which was preformed but not isolated, ${ }^{101}$

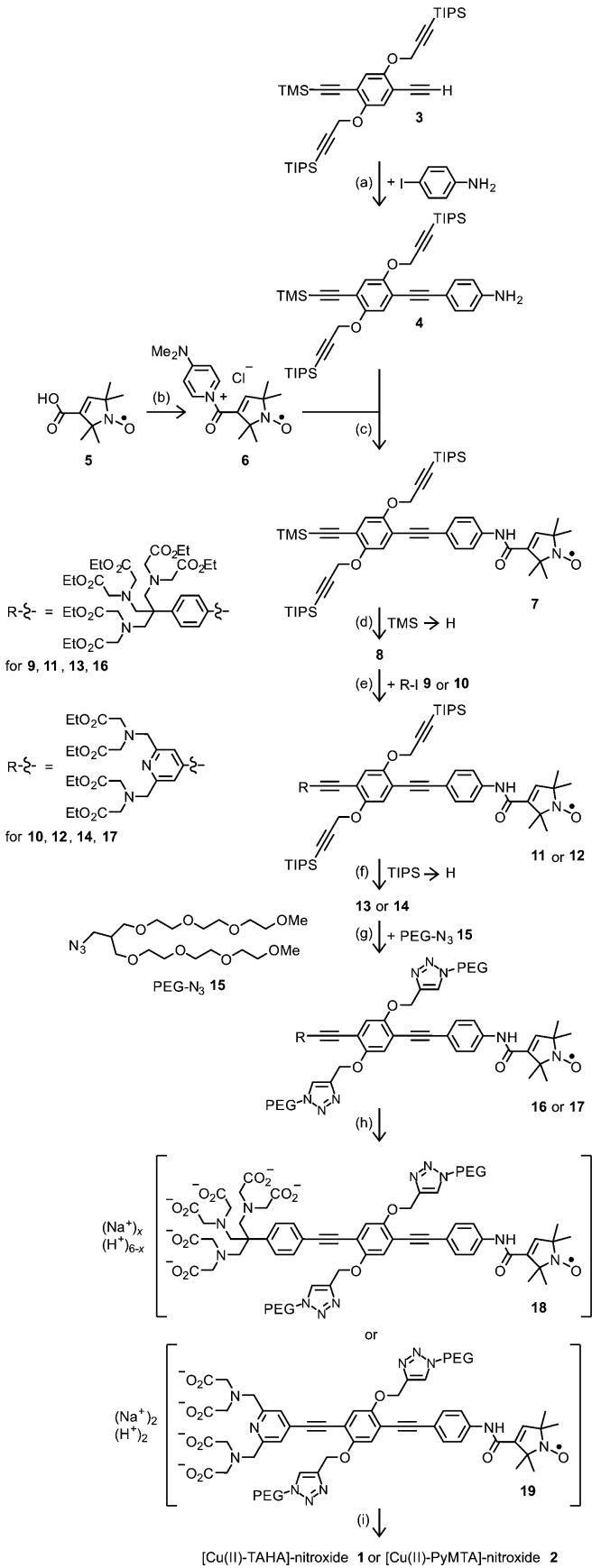

Fig. 3 Syntheses of the water soluble $\mathrm{Cu}(I I)$-nitroxide rulers 1 and 2 with $\mathrm{Cu}(I)-\mathrm{TAHA}$ and $\mathrm{Cu}(\|)-\mathrm{PyMTA}$ as the $\mathrm{Cu}(\mathrm{II})$-based spin labels. (a) $\mathrm{PdCl}_{2}\left(\mathrm{PPh}_{3}\right)_{2}$, Cul, piperidine, THF, rt, $25 \mathrm{~h}, 84 \%$; (b) (1) DMAP, $\mathrm{CH}_{2} \mathrm{Cl}_{2}$, (2) thionyl chloride, $0{ }^{\circ} \mathrm{C}$ to rt, 65 min; 6 was not isolated; (c) rt, 75 min, 89\%; (d) $\mathrm{K}_{2} \mathrm{CO}_{3}, \mathrm{MeOH}, \mathrm{CH}_{2} \mathrm{Cl}_{2}$, rt, 20.5 h, 99\%; (e): R-I 9, $\mathrm{PdCl}_{2}\left(\mathrm{PPh}_{3}\right)_{2}, \mathrm{Cul}^{\mathrm{i}} \mathrm{Pr}_{2} \mathrm{NH}, \mathrm{THF}, \mathrm{rt}, 23 \mathrm{~h}, 65 \%$ for 11; R-I 10, $\mathrm{Pd}\left(\mathrm{PPh}_{3}\right)_{4}, \mathrm{Cul}, \mathrm{Pr}_{2} \mathrm{NH}, \mathrm{THF}, \mathrm{rt}, 65 \mathrm{~h}, 53 \%$ for 12; (f) $\mathrm{Bu}_{4} \mathrm{NF}, \mathrm{THF}, 15$ min for 13; same reagents, 60 min for 14; (g) PEG- $\mathrm{N}_{3}$, [Cu(phen) $\left.\left(\mathrm{PPh}_{3}\right)_{2}\right] \mathrm{NO}_{3} \cdot 0.5 \mathrm{CH}_{2} \mathrm{Cl}_{2}$, toluene, rt, $94 \mathrm{~h}, 74 \%$ (2 steps) for 16; same reagents, rt, $41 \mathrm{~h}, 76 \%$ (2 steps) for 17; (h) (1) $\mathrm{NaOH}, \mathrm{H}_{2} \mathrm{O}$, rt, (2) $\mathrm{HCl}$ in $\mathrm{H}_{2} \mathrm{O}$, rt, $68 \%$ for 18 ; same conditions, $90 \%$ for 19; the average protonation degree of TAHA-nitroxide 18 is 1.7 protons per TAHA calculated with the $\mathrm{p} K_{\mathrm{a}}$ values reported for TAHA. ${ }^{83}$ The protonation degree at $\mathrm{pH} 7$ given with the formula of PyMTA-nitroxide $\mathbf{1 9}$ is based on the data for diethylenetriaminepentaacetic acid (DTPA) which is structurally similar to PyMTA. ${ }^{51}$ (i) $\mathrm{CuCl}_{2}, \mathrm{NaOD}, \mathrm{D}_{2} \mathrm{O}$, rt. For further details see $\mathrm{SI} 2$ (ESI $\dagger$ ). DMAP = 4-(dimethylamino)pyridine, phen $=$ phenanthroline, $\mathrm{THF}=$ tetrahydrofuran, TIPS = triisopropylsilyl, TMS = trimethylsilyl, $\mathrm{rt}=$ room temperature. 
gave the spacer-nitroxide conjugate 7 . In contrast to our work on dinitroxide rulers in which the nitroxide and the spacer were connected by an ester linkage, ${ }^{98}$ here an amide linkage was used because the ester linkage would have been cleaved when, on a later stage of the ruler synthesis, the PyMTA ethyl ester and TAHA ethyl ester are converted into the corresponding acids by alkaline ester hydrolysis. An acidic ester hydrolysis of the corresponding tert-butyl esters of the ligand moieties, which might be compatible with an ester bond between spacer and nitroxide, is excluded because the spacer backbone is harmed by trifluoroacetic acid, as experienced by us ${ }^{99}$ and others. ${ }^{102,103}$ After removal of the trimethylsilyl group from spacer-nitroxide conjugate 7 , the scaffold assembly was finalised with a SonogashiraHagihara coupling of spacer-nitroxide conjugate 8 with 4-iodoTAHA ethyl ester (9) and 4-iodo-PyMTA ethyl ester (10). Once again, polar tagging ${ }^{98,99,100}$ helped in isolation of the products (for details see SI2, ESI $\dagger$ ). $\mathrm{Cu}(\mathrm{I}$ ) is an essential catalyst for this coupling reaction. It is known that $\mathrm{Cu}(\mathrm{I})$ can reduce a nitroxide to the corresponding hydroxylamine. ${ }^{45,104}$ Indeed, hydroxylamine derived from the ruler precursor PyMTA-nitroxide 12 was isolated in a few percent yield $(<7 \%)$. In contrast, no hydroxylamine was detected in the crude product of the reaction between spacer-nitroxide conjugate 8 with 4-iodo-TAHA ethyl ester (9). Separation of hydroxylamine and nitroxide was achieved by preparative HPLC. In principle, a hydroxylamine could be oxidized to the nitroxide by just stirring the reaction mixture in air after the coupling is complete. ${ }^{104}$ However, PyMTA will get oxidized as well under these conditions, albeit comparatively slowly. ${ }^{105}$ To take no risk, the metal ions were trapped with a metal ion scavenger before exposing the reaction mixture to air. In the next step the triisopropylsilyl groups were removed to set free the terminal alkyne moieties for the $\mathrm{Cu}(\mathrm{I})$ catalysed alkyneazide cycloaddition with PEG-azide 15 for attaching the PEG side chains. Surprisingly, in this step no reduction of the nitroxide to the hydroxylamine was observed. Most likely the high stability of the copper(I) catalyst $\left[\mathrm{Cu}(\right.$ phenanthroline $\left.)\left(\mathrm{PPh}_{3}\right)_{2}\right] \mathrm{NO}_{3}$ against oxidation - the cycloaddition can be performed even in air $^{106}$ - prevents the reduction of the nitroxide. Saponification of the PEGylation products 16 and $\mathbf{1 7}$ gave the ligand-spacernitroxide conjugates TAHA-nitroxide 18 and PyMTA-nitroxide 19 ready for complexation. Adding copper(II) chloride and neutralising the aqueous solutions gave [Cu(II)-TAHA]-nitroxide 1 and [Cu(II)-PyMTA]-nitroxide 2. TAHA-nitroxide 18 and PyMTAnitroxide 19 were also used to prepare the water soluble rulers [Gd(III)-TAHA]-nitroxide and [M-PyMTA]-nitroxide with $\mathrm{M}=\mathrm{Gd}(\mathrm{III})$, Dy(III), and Mn(II). Rulers with the combination of nitroxide with these metal ions are important compounds for current research. ${ }^{42,43,46,84,85,88-91,107}$

\section{$3.2{ }^{2}$ H-ESEEM suppression in the RIDME experiments}

The RIDME experiments presented in this study were performed on the $\mathrm{Cu}(\mathrm{II})$-nitroxide rulers 1 and 2 in a glassy matrix of $\mathrm{D}_{2} \mathrm{O}$ and $\mathrm{d}_{8}$-glycerol. This resulted in moderately strong electron spin echo envelope modulations (ESEEM) in the RIDME traces at the deuterium nuclear Zeeman frequency $(\approx 7.8 \mathrm{MHz})$, which can interfere with the true $\mathrm{Cu}(\mathrm{II})$-nitroxide dipolar modulations, especially for short spin-spin distances. The performance of two methods for the suppression of ESEEM was tested on a 1:1 molar mixture of the nitroxide tempol (4-hydroxy-2,2,6,6-tetramethylpiperidinyloxyl) and the complex Cu(II)-TAHA 20 (see inset in Fig. $4(\mathrm{~A})$ ) in $\mathrm{D}_{2} \mathrm{O} / \mathrm{d}_{8}$-glycerol. This mixture (in the following referred to as 'reference sample') mimics the relaxation and ESEEM properties of the ruler solutions, yet the RIDME trace of this sample does not exhibit visibly resolved dipolar oscillations, and thus offers the opportunity to conveniently monitor the efficiency of an ESEEM-suppression method.

Recently, an averaging approach was presented ${ }^{77}$ in which the phase of the ESEEM modulations is shifted relative to that of the electron dipole-dipole contribution by step-wise incrementing the transverse evolution delays $t_{1}$ and $t_{2}$ (see Fig. 4(A), averaging scheme (a) and red traces). The averaging is performed by summation of traces where the increments are chosen to cover a full period of the dominating ESEEM frequency (here ${ }^{2} \mathrm{H}$ ). For our reference sample this averaging scheme led to an almost complete cancellation of ${ }^{2} \mathrm{H}$-ESEEM modulations in the RIDME traces using eight steps with an averaging increment of $d_{\mathrm{ES}}=16 \mathrm{~ns}$. The individual traces of an averaging cycle and the resulting summed trace are shown in Fig. S9 (ESI $\dagger$ ). We present here in addition a modified version of ESEEM averaging (scheme (b), and blue traces in Fig. 4(A)) which conserves the total transverse evolution period $t_{\text {tot }}=t_{1}+t_{2}$, by decrementing $t_{2}$ with the same step as the first delay $t_{1}$ is incremented. The resulting phase shift of the ESEEM
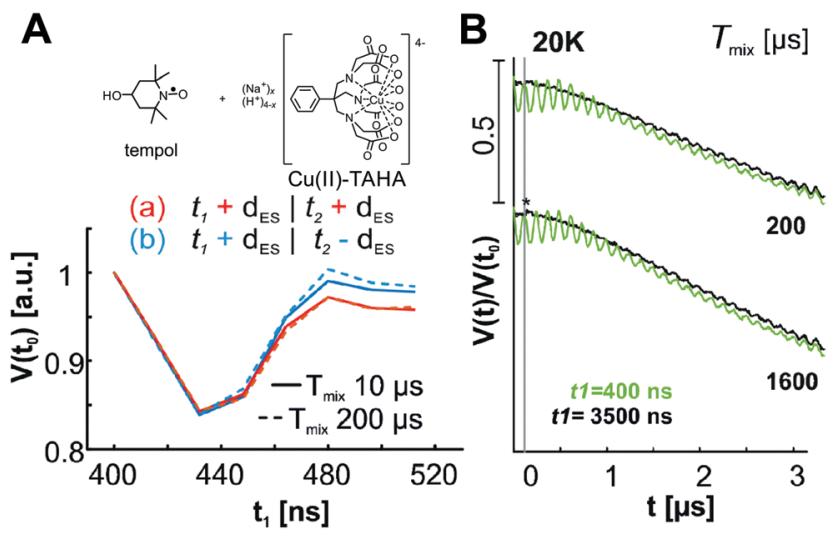

Fig. $4{ }^{2} \mathrm{H}$-ESEEM averaging approaches tested on a 1:1 molar mixture of tempol and [Cu(I)-TAHA] $20(100 \mu \mathrm{M}$ each, chemical structures given in the inset). For pulse stepping see red resp. blue arrows in Fig. 2(A). (A) The ${ }^{2} \mathrm{H}$-ESEEM averaging scheme presented in Keller et al. ${ }^{77}$ was tested for nitroxide-detected RIDME at two mixing times (red curves). The averaging occurs by simultaneously incrementing $t_{1}$ and $t_{2}$ with the step $d_{\mathrm{ES}}$. In this scheme the total experimental time $t_{\text {tot }}=t_{1}+t_{2}$ increases over a cycle, which leads to a minor signal decay. We found that a version with constant $t_{\text {tot }}$ performs equally well, while signal intensity is maintained (blue curves). (B) Primary RIDME data measured with short $t_{1}=400$ ns (green) and long $t_{1}=3500 \mathrm{~ns}$ (black) without additional ESEEM averaging for two $T_{\text {mix }}$ measured at $20 \mathrm{~K}$; the second refocusing delay was set to $t_{2}=3500 \mathrm{~ns}$ in both cases. ESEEM effects can be significantly suppressed by using a long $t_{1}$. This additionally suppresses a low-frequency component in the RIDME background (compare black and green traces at early times). ESEEM modulations are typically also reduced by using a long $T_{\text {mix }}$, but this did not significantly contribute to ESEEM suppression in these reference measurements. 
modulations is the same as in scheme (a), but the constant time experiment may be useful for efficient ESEEM averaging in samples with fast transverse relaxation.

In the course of this study we found yet another method to suppress ESEEM, which is based on increasing the first transverse evolution delay $t_{1}$ while keeping the second delay $t_{2}$ constant (compare green (short $t_{1}$ and black (long $t_{1}$ ) traces in Fig. 4(B))). Again the measurements were performed with the reference sample. All traces in Fig. 4(B) were acquired in the same amount of time, such that the SNR can be directly compared. In this particular case the reduction in SNR for the RIDME trace with longer transverse evolution time was low. The resulting suppression of ESEEM when using a long $t_{1}$, however, is striking. Note that this is most obvious for the easily recognisable ${ }^{2} \mathrm{H}$-ESEEM modulations, but in principle this suppression should apply to ESEEM arising from all relevant types of nuclei, because ESEEM modulations from all nuclei decay in accordance with the homogeneous linewidths and inhomogeneous broadening of the corresponding parts of the ESEEM spectrum. For RIDME detection on nitroxide radicals in water/glycerol mixtures, the ESEEM from ${ }^{1} \mathrm{H},{ }^{2} \mathrm{D}$, and ${ }^{14} \mathrm{~N}$ nuclei are of highest importance, which all have comparable characteristic ESEEM decay times. This is an advantage over the summationbased method, where the frequency of the unwanted ESEEM has to be known beforehand, in order to set up appropriate averaging delays. Furthermore there may be cases where the frequency of the dominating ESEEM contribution is very low, such as for example ${ }^{14} \mathrm{~N}$. In such cases even the constant-time averaging scheme reported in this work may not be sufficiently robust to enable averaging over one full period of the contaminating signal.

Of course, the two ESEEM suppression methods reported here do not mutually exclude each other, and a long $t_{1}$ can be combined with step-wise ESEEM averaging. We suggest the following empirical procedure for optimised ${ }^{2} \mathrm{H}$-ESEEM averaging: in our experience it is possible to find a $t_{1}$ that balances the loss of echo intensity against the amplitude of residual ESEEM contamination by measuring a 2-pulse (Hahn-echo) decay and setting $t_{1}$ to the value after which the deuterium ESEEM modulations in this Hahn-echo decay have visibly decayed. For additional experimental results (which may serve as a guide for efficient set-up of ESEEM suppression in solvents of varying degree of deuteration) we refer to the SI1 (ESI $\dagger$ ).

As a final remark in this section we want to point out that other methods for ESEEM averaging apart from the two presented in depth here have been devised and tested. ${ }^{37,59,86}$ These approaches are based either on dividing a given RIDME trace by reference data, recorded at the conditions of weak magnitude of the dipolar oscillations, or by reducing the excitation bandwidths of the m.w. pulses. Both of these approaches are costly in terms of SNR and were thus not further investigated in this study.

\subsection{RIDME background correction}

Correct evaluation of the intermolecular background contribution is an important step in any analysis of dipolar evolution data. The background decay in a RIDME experiment is typically steeper than the background decay in a DEER experiment, which can be mainly attributed to two reasons. First, for long mixing times the number of flipped B spins is rather large for the vicinity of each A spin, and, thus, the effective excitation probability for the RIDME mixing block is larger than for the typical DEER pump pulse. Accordingly, this results in a stronger intermolecular decay contribution in RIDME as compared to DEER. Second, there are nuclear spectral diffusion events during the RIDME mixing block, which are absent in the DEER experiment, where the mixing block is substituted by a pump pulse. The additional nuclear spectral diffusion makes RIDME background decay also steeper. Importantly, both electron spin flips and nuclear spectral diffusion depend on a number of factors (e.g. temperature, electron or nuclear spin concentration, spectral width), and thus the understanding of the RIDME background properties requires a dedicated study. Here, we only discuss few practical aspects, important for $\mathrm{Cu}(\mathrm{II})$-nitroxide RIDME.

The commonly applied empirical RIDME background shape is the stretched exponential decay function, ${ }^{29,76,86,108}$ but also second-order polynomial functions have been used for short dipolar evolution traces. ${ }^{28,49}$ We have found that for measurement temperatures of $30 \mathrm{~K}$ or lower, the use of the stretched exponential fitting function can lead to significant distortions in the dipolar spectra, as well as errors in the estimation of the modulation depth in the order of $10 \%$. In the case of the $\mathrm{Cu}(\mathrm{II})$-nitroxide rulers 1 and 2, the problem could be identified by looking at the mismatch between the RIDME form factor and the corresponding DeerAnalysis ${ }^{109} \mathrm{fit}$, at the time range of the first dipolar oscillation (see Fig. 5(A)). This effect can also be observed as a distortion of the dipolar spectrum (Fig. 5(B)). In the case of the $\mathrm{Cu}(\mathrm{II})$-nitroxide ruler 1 we do not observe a significant difference in the fitted distance distributions (Fig. 5(C)). For a short $t_{1}$ value $\left(t_{1}=400 \mathrm{~ns}\right.$ case in the Fig. $\left.5(\mathrm{~A})\right)$, a too deep first minimum in the dipolar oscillation is observed, which cannot be fitted by the standard DeerAnalysis kernel. We found that by increasing the first delay time to $t_{1}=3500 \mathrm{~ns}$, the shape of the primary RIDME trace had changed and a much better fit of the new form factor trace could be achieved.

This led us to investigate the shape of the $\mathrm{Cu}(\mathrm{II})$-nitroxide RIDME background function on a reference sample (1:1 molar mixture of $\mathrm{Cu}(\mathrm{II})$-TAHA 20 and the $\mathrm{Cu}(\mathrm{II})$-free TAHA-nitroxide 18, for chemical structures see Fig. 4, resp. Fig. 3). We used the ruler precursor molecules TAHA-nitroxide 18 instead of just adding nitroxide radicals to this sample to have nearly the same local surrounding on the nitroxide moiety in this reference sample and in the [Cu(II)-TAHA]-nitroxide ruler 1. At the same time, in such samples no metal ion exchange between $\mathrm{Cu}(\mathrm{II})-$ TAHA and $\mathrm{Cu}(\mathrm{II})$-free TAHA moieties in the rulers is expected, and, thus, any intramolecular effects due to $\mathrm{Cu}$ (II) are avoided. In this sample we saw that the RIDME background function rises first at short dipolar evolution times, before it begins to decay in the expected manner at longer times (see Fig. 5(D)). After background correction with a stretched exponential decay function (as one would do in a real RIDME experiment) this appears in frequency domain as a broad peak in the range of $0.5 \mathrm{MHz} \lesssim \nu_{\text {slow }} \lesssim 1.5 \mathrm{MHz}$ (Fig. 5(E)). This frequency range lies below any Q-band ESEEM frequency of commonly occurring nuclei. We also observed this behaviour of the RIDME background 

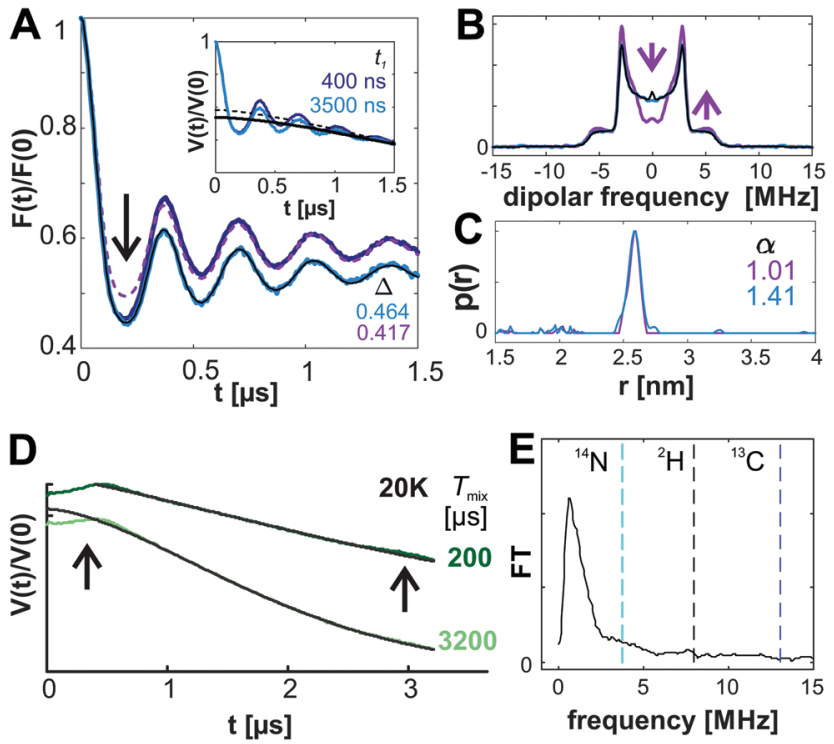

Fig. 5 (A) RIDME primary data (inset) and form factor recorded with $t_{1}=400 \mathrm{~ns}$ (purple in panels $(\mathrm{A}-\mathrm{C})$ ), resp. $t_{1}=3500 \mathrm{~ns}$ (blue in panels (A-C)) recorded at $T=20 \mathrm{~K}$ with $T_{\text {mix }}=400 \mu$ s on [Cu(॥)-TAHA]-nitroxide 1. The slowly oscillating background component in measurements with short $t_{1}$ can lead to incorrect background fitting (purple trace, and purple dashed line fit in inset), and consequently to significant errors in the estimation of the modulation depth $(\approx 10 \%)$. Little agreement of form factor fit with the experimental data early in the time-trace is an indication for this artifact. We found that increasing $t_{1}$ reduces the intensity of this artifact, which leads to a better background fit with a stretched exponential (blue trace and black solid line). (B) Distortions of the dipolar spectrum are observed, which are similar in appearance to orientation selection. (C) The effect on distance analysis of narrow distributions, where strong modulations are observed, is negligible. (D) The low frequency artifact (indicated by arrows) is clearly visible in background measurements obtained with a 1:1 molar mixture of TAHA-nitroxide 18 and [Cu(II)-TAHA] 20 (100 $\mu \mathrm{M}$ each). It cannot be eliminated by using longer $T_{\text {mix }}$ ( $(E)$ The dominating frequency from FT after background correction with a stretched exponential is lower than the expected ESEEM frequencies of common nuclei. Note that a weak contribution in the range of ${ }^{14} \mathrm{~N}$-ESEEM is also observed.

with samples of only the nitroxide tempol, which excludes effects originating from the presence of $\mathrm{Cu}$ (II) spins, or the molecular ruler (see Fig. S13, ESI $\dagger$ ). Based on these observations we can assume that the background artefact is not due to a direct ESEEM effect of any particular nucleus, or a certain specific group of nuclei, and it is related to the detection on organic radicals and is independent on the presence or absence of other paramagnetic centres, such as $\mathrm{Cu}$ (II) ions. Empirically, we found that the overall background shape can be fitted with a sum of two stretched exponential functions, so that the artefact intensity in the very low frequency range is suppressed to an acceptable extent, allowing for a good quality fit of the form factor data, within the noise level (see Fig. S17, ESI $\dagger$ ). Such fitting is possible for samples with a narrow distance distribution, where one or more clearly visible dipolar oscillations can be observed in the RIDME trace. For samples with a broad distance distribution, however, this is expected to be unstable, because the rising RIDME background component and the intramolecular contributions may overlap and cannot easily be distinguished from each other.
More generally, this background artefact would cause a problem of accurate determination of any form factor which does not contain clear dipolar oscillations. Besides broad distance distribution, this can also arise from a distribution of spin-spin exchange couplings or, in some cases, even from the orientation selection effects.

The extent to which the background shape deviates from a single stretched exponential depends on the duration of the first transverse evolution period. Increasing the first refocusing delay $t_{1}$ suppresses the low-frequency contamination in the background traces (see Fig. 4(B)). If $t_{1}$ is chosen sufficiently long, a stretched exponential background shape is recovered. With this choice of experimental parameters the background fitting procedure is also considerably more stable with respect to the choice of fit range (see Fig. S15, ESI $\dagger$ ). More experimental results and a recommended procedure to find pulse delay settings that recover a stretched exponential background shape are given in the SI1 (ESI $\dagger$ ).

In retrospect, it is apparent that this background feature is present as well in previously published metal ion-nitroxide RIDME data. ${ }^{29,49}$ In some other cases reported in the literature a different background fitting methods were applied, which makes direct comparison without the primary data difficult. For previously reported metal-metal RIDME data this problem is either absent or too weak to be clearly detected. ${ }^{76}$

When varying the degree of the solvent deuteration, we oberved the expected trend that an increase of the proton concentration leads to significantly steeper RIDME background decay. This smears out the discussed background distortion, but in our measurements in most cases it is still not described accurately by a stretched exponential function (see Fig. S13(C), $\mathrm{ESI} \dagger$ ). In our experience, for a nitroxide sample in fully deuterated solvent a stretched exponential background shape could be observed with a $t_{1}$ value of about $3500 \mathrm{~ns}$, while for a sample with $15 \% \mathrm{H}_{2} \mathrm{O}$ the minimal required $t_{1}$ value could be reduced to $1500 \mathrm{~ns}$ (see Fig. S13, ESI $\dagger$ ). Interestingly, the typical time $t_{1}$ at which the RIDME background distortion is suppressed is comparable to the typical ESEEM dephasing times in the two and three pulse ESEEM experiments on the same samples.

Given the presented set of RIDME background measurements we cannot draw a firm conclusion on the mechanism that causes the deviation from a stretched exponential decay. It may arise due to some dephasing process, which is relevant only at short $t_{1}$ times, but disappears at long $t_{1}$. We remark that at low concentrations (of 100 to $200 \mu \mathrm{M}$ ) it is likely that nuclear-driven mechanisms are the dominating ones for the RIDME background decay. Instantaneous diffusion processes are unlikely causes, because we have observed the background artifact both in traces measured with soft (50/100 ns) and hard (12/24 ns) pulses.

While the discussed background artefact cannot be due to some particular nucleus or group of nuclei, and it does not contain any characteristic nuclear Zeeman frequency, there is still a feasible mechanism for such a signal to appear from ESEEM. The joint action of the primary Hahn spin echo and the composite $\pi$-pulse formed by the two $\pi / 2$-pulses of the mixing 
block produces an ESEEM signal similar to the one in the well known two-pulse ESEEM experiment. This signal must contain contributions, oscillating with the sum and the difference of the nuclear frequencies in the $\alpha$ - and $\beta$-manifold of the electron spin. For weak hypefine couplings of the order of 1-2 $\mathrm{MHz}$, which would correspond to the characteristic frequencies of the background artefact (see Fig. S17, ESI $\dagger$ ), the difference frequency would essentially contain only the secular part of the hyperfine coupling tensor, while the nuclear Zeeman frequency would be removed due to the subtraction. The absence of such a background artefact in the metal-metal RIDME would then be explained by the smaller number of intramolecular proton couplings, by the larger average electron-proton distances, and by a more symmetric distribution of the intramolecular protons around the paramagnetic ion. This would mean that the background artefact is also present in the metal-metal RIDME data, but that it is too weak to be unambiguously detected. The time dependence of such an ESEEM artefact, estimated on the basis of the two-pulse ESEEM theory, would be consistent with both positive and negative magnitude of this contribution in the RIDME background, depending on a particular geometry, time delays, and orientation-dependent signs of the hyperfine couplings. If the couplings are weak, the described ESEEM contribution would not be much suppressed by the reduced pulse bandwidth of the $100 \mathrm{~ns}$ pump pulse, as experimentally observed and described above. The only fundamental issue of such a mechanism is that such a difference ESEEM frequency should appear along with the proton nuclear Zeemen frequency contribution and the contribution with doubled proton Zeeman frequency, which are weak in the presented experiments.

Further understanding of this mechanism will be of interest in future studies. However, for practical reasons, the increase of $t_{1}$ appears to be a sufficiently good work-around for this problem in cases where some loss of RIDME signal intensity can be tolerated. In the presented here case an increase of $t_{1}$ sufficient to suppress this background artefact resulted in approximately $20 \%$ sensitivity loss. This work-around is essential in studies of samples with broad spin-spin distance distributions, which feature dipolar evolution traces without easily recognisable oscillations. For such samples the RIDME background decay artefact would not easily be distinguishable from the true dipolar signal.

Importantly, the division approach, ${ }^{86}$ which is often used for the ESEEM artefact reduction is also capable to suppress this background problem to an acceptable level (see details in the SI1, ESI $\dagger$ ). In the situations when transverse relaxation of nitroxide radicals through the sample is approximately the same, the use of the proposed here elongation of $t_{1}$ appears more advantageous, due to the better overall sensitivity. However, if detected spins have a broad distribution of relaxation times, use of long $t_{1}$ might result in some relaxation filtering effects, and thus the division approach to remove the background artefact might appear more advantageous despite its higher sensitivity loss.

Note also that we have checked that the described here background artefact is not related to any echo crossing. This is in line with the artefact's widths, which is much larger than for any echo crossing feature we observed in these RIDME data.

\subsection{Echo-crossing artifacts}

The originally reported eight-step phase cycling protocol ${ }^{59}$ includes two-step $[+(+x)-(-x)]$ phase cycling on the first $\pi / 2$ pulse and four-step $[+(+x)+(+y)+(-x)+(-y)]$ phase cycling on the two $\pi / 2$ pulses from the RIDME mixing block, with the same phase of the two pulses in each phase cycling step. In particular, this phase cycling protocol is designed to remove the echo crossing artefact due to the refocused stimulated echo (all five pulses act) and the two-pulse Hahn echo from the last two pulses of the RIDME pulse sequence. Together with longitudinal relaxation of the A spins, imperfections in the channel balance of the pulse EPR spectrometer lead to incomplete removal of the latter echo crossing artefact, so that a peak is observed at approximately zero time in the RIDME trace, as discussed previously. ${ }^{76}$ From plotting the echo transients of a RIDME experiment we also see that at very long mixing times the primary echo from the last two pulses becomes much stronger than the RIDME RVE echo, and results in an artificially high modulation depth, as will be discussed in the following section. This has been observed as well for Gd(III)-Gd(III) RIDME. ${ }^{76}$

For a more general approach to monitor echo crossings, we computed a list of coherence order pathways and resulting echos following the well-established concepts reported in gemperle et al. ${ }^{110}$ where we considered only terms of coherence orders $-1,0$, and 1 . This identification of echos is potentially helpful to assign echo-crossing artifacts for any arbitrary choice of experimental parameters $\left(t_{1}, t_{2}, \ldots\right)$. For $N_{\mathrm{p}}=5$-pulse RIDME this amounts to $3^{N_{\mathrm{p}}-1}=81$ pathways. By nature of the detection method, however, we only needed to consider pathways that end up in coherence order -1 after the final pulse. In addition, we assumed that all coherence pathways, which are not in coherence order 0 during the mixing block, are strongly attenuated due to transverse relaxation, and can be neglected. With this approximation we arrived at 27 relevant pathways, for which the full list is given in Table S3 (ESI $\dagger$ ). The refocusing condition as well as the time-step with which the echo moves with respect to the stationary RIDME RVE echo for all identified echos is also given in the SI1 $(\mathrm{ESI} \dagger)$. Note that from these values it is possible to calculate the echocrossing point for an arbitrary choice of delays $t_{1}, t_{2}$ and time step $\mathrm{d} t$, which may be useful in the identification of echo crossing artefacts in general for 5-pulse RIDME experiments. An example is calculated in the SI1 (ESI $\dagger$ ). To determine which echo pathways are relevant in a real RIDME experiment we detected echo transients (without phase cycling) for several time-points. We found that for mixing times in the recommended range (see next section) we can assign all experimentally relevant echo traces in the detection window to our calculated echo pathways, which validates our assumption that the remaining pathways are strongly attenuated in nitroxide- $\mathrm{Cu}$ (II) RIDME during $T_{\text {mix }}$ (compare Fig. S22, ESI $\dagger$ ). Importantly, we can see that an echo-crossing is not the cause for the low-frequency artifact reported above, as is discussed in detail in the SI1 (ESI $\dagger$ ).

For the setting $t_{1}=t_{2}$, there is a stimulated echo from the first, second and fifth pulse (RIDME block does not act), which does not shift when stepping the mixing block, and which is not 
removed by the standard eight-step phase cycling. This echo is not modulated with dipolar frequencies as a function of the mixing block position $t$, but its presence leads to an apparent reduction of the dipolar modulation depth. This echo should be removed for example by the phase cycle: $[+(+x)-(-x)]$ on the first $\pi / 2$ pulse, $[+(+x)+(+y)+(-x)+(-y)]$ on the two $\pi / 2$ mixing block pulses and $[+(+x)-(+y)+(-x)-(-y)]$ on the two refocusing $\pi$ pulses (see also SI1, ESI $\dagger$ ). Measuring at $t_{1}=t_{2}$ was not extensively tested, but may present interesting conditions, as this represents a case of a dynamically decoupled pulse sequence.

\subsection{Modulation depth build-up and optimal measurement temperature}

If we consider a Poisson stochastic flipping process in a twolevel spin system, characterised by the flip rate $W$, it is possible to show ${ }^{111}$ that the probabilities for the even and the odd number of spin flips $N_{\text {flip }}$ after a time $T_{\text {mix }}$ are given by

$$
P_{\text {even }}=\frac{1}{2}\left(1+\exp \left(-2 W T_{\text {mix }}\right)\right)
$$

and

$$
P_{\text {odd }}=\frac{1}{2}\left(1-\exp \left(-2 W T_{\text {mix }}\right)\right)
$$

The $\mathrm{Cu}(\mathrm{II})$ ion as well as the nitroxide has an electron spin of $S=1 / 2$ with two spin states. Only those B spins contribute to dipolar modulation that undergo an odd number of spin flips during the mixing block. Thus, the build-up kinetics for the RIDME modulation depth follows the law for $P_{\text {odd }}\left(T_{\text {mix }}\right)$. We can assign $2 W=T_{1, \mathrm{~B}}$, where $T_{1, \mathrm{~B}}$ is the longitudinal relaxation time of the B spins. This approximation for the RIDME modulation buildup kinetics has been used in the past. ${ }^{112}$
This approximation neglects that longitudinal relaxation of paramagnetic centres in frozen glasses is typically not monoexponential and that the B-spin flip may induce a flip of the A spin with certain probability. Such processes are of relevance in Gd(III)-Gd(III) RIDME ${ }^{76}$ and might play a role in $\mathrm{Cu}(\mathrm{II})$-nitroxide RIDME as well. Within the approximation $(1,2)$, the dipolar modulation amplitude in the RIDME experiment grows with a characteristic rate of $1 / T_{1, \mathrm{~B}}$ and reaches a steady state value of $50 \%$ of the total spin echo amplitude after a time $T_{\text {mix }} \gg T_{1, \mathrm{~B}}$. The neglected correlated flip-flops of the A-B pairs can lead to a reduced limiting value of the modulation depth, since the average number of B spin flips for the modulated part of the spin echo signal is always larger than the average number of $\mathrm{B}$ spin flips for the unmodulated part of the spin echo.

The dipolar modulation builds up during the mixing time due to stochastic flips of the $\mathrm{B}$ spins on a $T_{1, \mathrm{~B}}$ time scale. Longitudinal relaxation of the A spins must be sufficiently slow, so that the non-equilibrium A-spins magnetisation survives during $T_{\text {mix }}$. While this can become a problem for metal-metal RIDME, where the A and B spins have the same relaxation rates, the condition is easily met for metal ion-nitroxide RIDME. For the $\mathrm{Cu}$ (II)-nitroxide rulers studied here, we found that at any tested measurement temperature, longitudinal relaxation of nitroxides $T_{1 \mathrm{e} \text {,NO }}$ was much slower than that of the $\mathrm{Cu}(\mathrm{II})$ ions. This is expected to be typical for $\mathrm{Cu}(\mathrm{II})$-nitroxide spin pairs. Therefore it is indeed possible to use a mixing time $T_{\text {mix }} \gg T_{1, \mathrm{~B}}$ without significantly attenuating the echo signal of the nitroxide.

Suitable conditions for the orthogonal RIDME experiment in the $\mathrm{Cu}(\mathrm{II})$-nitroxide spin pair were identified by screening temperature and mixing time for $[\mathrm{Cu}(\mathrm{II})-\mathrm{TAHA}]$-nitroxide 1 . The primary data of RIDME measurements at $30 \mathrm{~K}$ are shown in Fig. 6(A) and the corresponding distance distributions obtained with
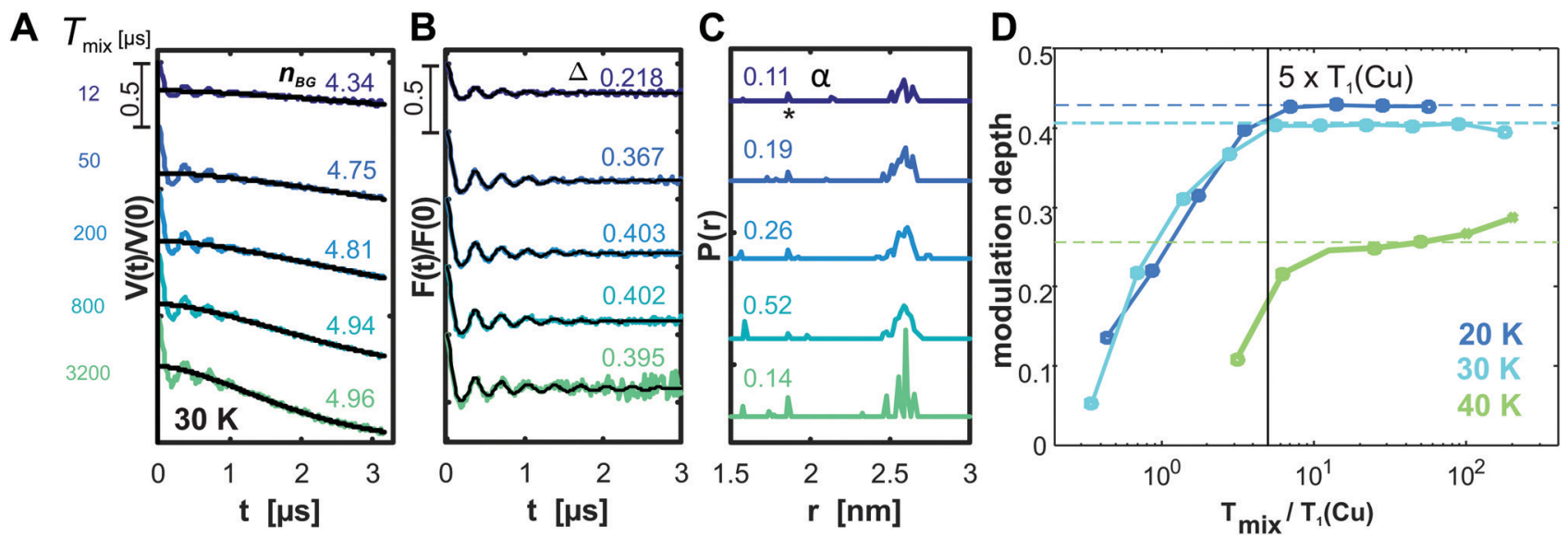

Fig. $6 T_{\text {mix }}$ screening on [Cu(II)-TAHA]-nitroxide 1 measured at $30 \mathrm{~K}$ (without ${ }^{2} \mathrm{H}$-ESEEM averaging, $t_{1}=3500$ ns). Data were analysed using the DeerAnalysis software package ${ }^{109}$ with an automatic choice of the regularisation parameter $\alpha$. (A) Primary data, shifted for clarity; (B) form factors after stretched exponential BG correction; (C) distance distributions; the dimensionality of the background $n_{\mathrm{BG}}$ increases with $T_{\text {mix. }}$. At very long $T_{\text {mix }}$ the BG decay becomes so steep, that the dipolar modulations are partially suppressed. The artifact contribution in the distance distribution from the ${ }^{2} \mathrm{H}$-ESEEM contamination is indicated in one trace by an asterisk. The SNR in this screening series is not yet optimal for distance analysis, which leads to slight overfitting of the data. (D) The observed $\lambda$ in the series shown in (A-C), as well as analogous results for different temperatures is shown as a function of the ratio of $T_{\text {mix }}$ and the characteristic time of longitudinal relaxation of $\mathrm{Cu}(\mathrm{II}), \mathrm{T} 1(\mathrm{Cu})$, for three different temperatures. At all temperatures the modulation depth reaches a plateau (dashed lines). The data points marked with an ' $x$ ' are derived from experiments that are contaminated by an echo crossing-artifact, which artificially increases the observed $\lambda$. The solid vertical line indicates the corner of the plateau, which for all temperatures occurs approximately at $5 \cdot T_{1(\mathrm{Cu})}$. 
DeerAnalysis $2016^{109}$ using Tikhonov regularisation are displayed in Fig. 6(B) (see Fig. S8 for an example of data processing, ESI $\dagger$ ). Deviations of the background decay functions from the stretched exponential function characteristic for short $t_{1}$ delay times resulted in uncertainties of the determined modulation depths (Fig. S16, ESI $\dagger$ ). Accordingly, analysis of the modulation depth was performed using RIDME measurements with long $t_{1}$ times. The results for the modulation depth values are shown in Fig. 6(D). No nitroxide orientation averaging scheme was used in this series (primary data and form factors in the series displayed in Fig. S20, ESI $\dagger$ ).

For all tested temperatures we found that the modulation depth reaches a plateau when applying long mixing times $T_{\text {mix }} \approx 5 \cdot T_{1, \mathrm{Cu}}$. The value of the modulation depth at this plateau $\lambda_{\max }(T)$ is given in Table 2 and is higher at lower temperatures. Nonetheless, even at $20 \mathrm{~K}$ the limiting value is about $10 \%$ lower than the theoretical limit of 0.5. There might be at least two possible contributions to this loss of modulation depth. First, as indicated also by the measurements at higher temperatures, a non-negligible contribution from the A-spin relaxation induced by the B-spin flips might be present. Second, we cannot exclude incomplete loading of the ligand with $\mathrm{Cu}(\mathrm{II})$ as an alternative explanation of the lower modulation depth. These two effects can also interfere, because A-spins which are not in close proximity to a B-spin do not experience this flip enhancement, and would thus be over-represented in the detected echo. Note that the same effect was discussed for high-spin Gd(III)-Gd(III) and Mn(II)-Mn(II) RIDME, where, besides the modulation depth reduction, it also affects the buildup kinetics for different dipolar frequency overtones. ${ }^{77}$ Longitudinal relaxation of the nitroxide spins is extremely slow at $10 \mathrm{~K}$, enforcing a low shot repetition rate. This severely reduces sensitivity and we did not study RIDME modulation buildup in detail at this temperature. At $40 \mathrm{~K}$ we found that the extracted value of the modulation depth increases again after the plateau (data points marked as crosses). This apparent additional modulation is a consequence of the incomplete cycling of a crossing echo from the last two pulses $(\pi / 2-\tau-\pi$ sub-sequence) with opposite phase and must not be interpreted as additional dipolar modulation. This can also be seen in the form factor, where a steep initial decay of the trace is observed, which leads to artifact distance peaks at very short distances. Further demonstration of the effect of this echo-crossing artifacts can be found in Fig. S19(B) (ESI $\dagger$ ).

The best results were obtained at $20 \mathrm{~K}$ and $30 \mathrm{~K}$, with mixing times ranging from several hundreds of microseconds to several milliseconds $T_{\text {mix,opt }} \approx 400 \mu \mathrm{s}-1.6 \mathrm{~ms}$. This is much longer than the phase memory time of the detected nitroxide spins $T_{\text {mix opt }} \approx 50-100 \cdot T_{\mathrm{m}, \mathrm{NO}}$. For the choice of mixing times within the range of the modulation depth plateau we observe that ESEEM contributions, which can still be comparatively strong at short mixing times, are suppressed by going to very long $T_{\text {mix }}$. This advantageous trend is counteracted by a clear loss of SNR, when $T_{\text {mix }}$ approaches $T_{1, N O}$ for the given temperature. This is also the range, where the echo-crossing artifact contamination at zero-time becomes increasingly strong.

We determine the optimal measurement temperature for a sample given its relaxation properties following the argumentation for the optimisation of nitroxide-nitroxide DEER in Jeschke and Polyhach $^{113}$ Note that we expect this optimisation to hold for a wide range of samples, if the relevant relaxation properties are known. The rationalisation for the individual terms is given in the SI1 (ESI $\dagger$ ), here we only show the final equation that was used for the optimisation. We define the quantity $S_{r_{\max }}$

$$
\begin{aligned}
S_{r_{\text {max }}}(T) \approx & \frac{1}{T} \cdot \exp \left(-5 T_{1, \mathrm{Cu}} / T_{1, \mathrm{NO}}\right) \cdot \exp \left(-2 t_{r_{\max }} / T_{m, \mathrm{NO}}\right) \\
& \cdot \sqrt{T_{\mathrm{ref}} / T_{1, \mathrm{NO}}} \cdot \lambda_{\max }(T)
\end{aligned}
$$

which takes into account that the expected optimal experimental conditions depend on the longest expected distance $r_{\max }$, which manifests in a required minimum dipolar evolution trace length $t_{r_{\text {max }}}$. The optimisation is in terms of the temperature $T$, the longitudinal relaxation times of the two spins $T_{1, \mathrm{Cu}}$ and $T_{1, \mathrm{NO}}$, the transverse relaxation of time of the detected nitroxide spins $T_{m, \mathrm{NO}}$, and the empirical maximal modulation depth plateau value $\lambda_{\text {max }}(T)$. The factor $\sqrt{T_{\text {ref }} / T_{1, \text { NO }}}$ accounts for the fact that at lower temperatures it is necessary to use lower shot repetition rates to avoid saturation of the detected spin, and $T_{\text {ref }}$ is an arbitrary reference time to obtain a dimensionless parameter. In eqn (3) we have already inserted $T_{\text {mix }}=5 \cdot T_{1, \mathrm{Cu}}$, which corresponds to the point where the modulation depth plateau is reached at all tested temperatures. The transverse and longitudinal relaxation properties for nitroxide spins and $\mathrm{Cu}$ (II) complexes at the relevant temperatures were measured and the primary data can be found in the SI1 (ESI $\dagger$ ). The experimental 1/e times for transverse relaxation resp. the $(1-1 / e)$ times for inversion recovery are summarised in Table 1. Note that $\mathrm{Cu}$ (II) complexes feature weak anisotropy of the longitudinal relaxation time (see Fig. S7, ESI $\dagger$ ), which we neglect in the following.

The $S_{r_{\max }}$ for the tested temperature range is calculated using this equation from the values reported in Tables 1 and 2 . The results are normalised to $S_{r_{\max }}$ at $20 \mathrm{~K}$, since the absolute values are not relevant for temperature optimisation and depend on the arbitrary choice of $T_{\text {ref }}$. This allows us to use $S_{r_{\max }}$ as given in Table 2 to state the following recommendation: depending on the expected spin-spin distances, the best measurement temperature slightly varies. For distances below $4 \mathrm{~nm}$ we recommend an experimental temperature between $30 \mathrm{~K}$ and $40 \mathrm{~K}$. The modulation

Table 1 Relaxation properties of [Cu(॥)-TAHA]-nitroxide 1 in the temperature range 10-50 K; fits of the data with different models can be found in the SI1 (ESI). Here we report the delays until a given fraction of spins has relaxed

\begin{tabular}{|c|c|c|c|c|c|}
\hline & $10 \mathrm{~K}$ & $20 \mathrm{~K}$ & $30 \mathrm{~K}$ & $40 \mathrm{~K}$ & $50 \mathrm{~K}$ \\
\hline (IR) signal recovered to & \multicolumn{5}{|c|}{ Nitroxide $[\mathrm{ms}]$} \\
\hline $1-1 / e \approx 0.63$ & \multicolumn{5}{|c|}{$\mathrm{Cu}(\mathrm{II})(\operatorname{maximum})[\mu \mathrm{s}]$} \\
\hline $1-1 / e$ & 2022 & 57 & 18 & 2 & 1 \\
\hline$\left(T_{\mathrm{m}}\right)$ signal decayed to & \multicolumn{5}{|c|}{ Nitroxide $[\mu s]$} \\
\hline $1 / e \approx 0.37$ & $\begin{array}{l}8.7 \\
\mathrm{Cu}(\mathrm{II})\end{array}$ & \multicolumn{3}{|c|}{$\mathrm{Cu}(\mathrm{II})$ (maximum) $[\mu \mathrm{s}]$} & 3.2 \\
\hline $1 / e$ & 7.7 & 6.7 & 4.2 & 2.4 & 1.7 \\
\hline
\end{tabular}
towards equilibrium (indicated as dashed lines in Fig. S3-S6, ESI) 
Table 2 Optimisation of experimental temperature based on the nitroxide and $\mathrm{Cu}(I)$ relaxation properties at different temperatures and the observed maximum modulation depth. The values for $S_{r_{\max }}$ were calculated using eqn (3) for three values of $r_{\max }$ with the values given in Table 1 and are given relative to $S_{r_{\max }}$ at $20 \mathrm{~K}$. $\lambda_{\max }$ is tabulated as well and is the value of the modulation depth at the plateau reported in Fig. 6(C)

\begin{tabular}{llll}
\hline & $20 \mathrm{~K}$ & $30 \mathrm{~K}$ & $40 \mathrm{~K}$ \\
\hline$\lambda_{\max }(T)$ & 0.429 & 0.406 & 0.248 \\
$S_{r_{\max }}(T) / S_{r_{\max }}(20 \mathrm{~K})$ & & & \\
$r_{\max }=2 \mathrm{~nm}$ & & & \\
$r_{\max }=4 \mathrm{~nm}$ & 1 & 2.6 & 3.4 \\
$r_{\max }=6 \mathrm{~nm}$ & 1 & 2.4 & 2.7 \\
\hline
\end{tabular}

depth may still be increased (due to higher $\lambda_{\max }$ at low temperatures), but no benefit for sensitivity is expected from going to lower temperatures due to the slow longitudinal relaxation of the nitroxide spins. To measure distances above $4 \mathrm{~nm}$ it may be better to measure at the lower limit ( $30 \mathrm{~K}$ ), but again, even lower temperatures (tested: $20 \mathrm{~K}$ ) are not expected to increase sensitivity.

We tested on [Cu(II)-TAHA]-nitroxide 1 that detection of RIDME time traces in the regime of partial nitroxide saturation (due to too fast repetition rates) does not change the shape of the dipolar oscillations (see Fig. S10, ESI $\dagger$ ). Adding a $T_{1}$-relaxation enhancer ${ }^{114}$ might help to improve the longitudinal relaxation properties of nitroxide spins in these experiments, however, this strategy may not generally be viable for biological samples. For short spin-spin distances, the length of the RIDME time traces is not very critical, and one can optimise the modulation depth and mixing time, which is achieved at somewhat higher temperature. Note that our estimate agrees very well with the RIDME measurement temperatures used for [Cu(II)-terpyridine]-nitroxide. ${ }^{28,29}$

Our treatment neglects longitudinal relaxation of $\mathrm{Cu}$ (II) spins during the time where A spin magnetisation is in the transverse plane, which may lead to a loss of the dipolar modulation depth. Such a case was recently reported for room-temperature RIDME measurements in trityl-nitroxide pairs. ${ }^{115}$ Note that for unequal A and $\mathrm{B}$ spins it might be even possible to reach conditions under which longitudinal relaxation of B spins is much faster than the transverse relaxation of the A spins, and the dipole-dipole interaction is thus averaged.

We also neglect that the dimensionality of the background decay changes with the mixing time due to a prolonged period of spectral diffusion. This decay can eventually become so steep, that part of the dipolar oscillations are suppressed, which leads to an artificial broadening of the distance distribution (see Fig. 6(C)). In cases of very fast background decay it may be necessary to use mixing times shorter than optimal according to eqn (3). This may be partially compensated by raising the experimental temperature, where higher modulation depths can be achieved for the same absolute mixing time.

\subsection{Orientation-resolved and orientation-averaged $\mathrm{Cu}(\mathrm{II})$-nitroxide RIDME with rectangular pulses setup}

Both $\mathrm{Cu}(\mathrm{II})$ and nitroxide are electron spin $1 / 2$ species and for the $\mathrm{Cu}(\mathrm{II})$-nitroxide rulers $\mathbf{1}$ and 2, the two types of paramagnetic species have rather large resonance frequency offsets. Thus, only the secular part of the electron-electron dipole interaction needs to be considered. The analysis of dipolar evolution time-traces $V(t)$ for pairs of such spins in a homogeneous frozen glassy sample can be interpreted with the conventional point-dipole approximation for dipole-dipole coupling. This model relates the distance between the two spins $r$ to a dipolar frequency $d(r, \theta)$ which depends on the angle of the inter-spin vector to the external magnetic field

$$
d(r, \theta)=\omega_{\perp}(r)\left(1-3 \cos ^{2}(\theta)\right)
$$

where $\omega_{\perp}$ is the dipolar frequency for perpendicular orientation of the inter-spin vector to $B_{0}$.

The extraction of distance distributions from pulsed dipolar experiments relies on the dependence of the parameter $\omega_{\perp}$ on the inter-spin distance $r$ :

$$
\begin{gathered}
\omega_{\perp}(r)=\frac{\mu_{0} \beta_{\mathrm{e}}^{2}}{4 \pi \hbar} \frac{g_{\mathrm{A}} g_{\mathrm{B}}}{r^{3}} \\
\approx \frac{52.04 \mathrm{MHz}}{(r / 1 \mathrm{~nm})^{3}}
\end{gathered}
$$

What is typically neglected for nitroxides and other organic radicals is the deviation of the $g$ factor of the radical from that of the free electron, thus $g_{\mathrm{A}}=g_{\mathrm{B}} \approx g_{\mathrm{e}}$, which results in the approximation given in eqn (6), which is made in the kernelgenerating function of DeerAnalysis. ${ }^{109}$ In the $\mathrm{Cu}(\mathrm{II})$-nitroxide RIDME case, this approximation is very good for $g_{\mathrm{A}}=g_{\mathrm{NO}}$, but for $\mathrm{Cu}$ (II) as the coupled spin, deviations can arise for $g_{\mathrm{B}}=g_{\mathrm{Cu}}$. For the case of $\mathrm{Cu}(\mathrm{II})$ complexes with known $g$ factors, an approximate correction has been previously reported. ${ }^{116}$ More information on this can be found in the SI1 (ESI $\dagger$ ). For the often observed approximately square planar coordination of $\mathrm{Cu}$ (II), the largest deviation of $g_{\mathrm{Cu}}$ from $g_{\text {free }}$ is along the $z$-axis and, as an example, a value of $g_{\mathrm{Cu}, z}=2.2$ leads to a deviation of $\omega_{\mathrm{dd} \text {,eff }}$ of $100 \% \cdot\left(g_{\mathrm{B}}-g_{\mathrm{e}}\right) / g_{\mathrm{e}}=9.9 \%$. This translates to an error in the distance calculation of $3.2 \%$. In this work, we neglect such effects and use the standard kernel implemented in DeerAnalysis ${ }^{109}$ to process the pulse EPR data.

Orientation-resolved $\mathrm{Cu}(\mathrm{II})$-nitroxide RIDME data were obtained by measuring a series of dipolar evolution time traces at different positions in the nitroxide spectrum. We used a $\pi / 2$ pulse length of $50 \mathrm{~ns}$ and a $\pi$ pulse length of $100 \mathrm{~ns}$ in these experiments in order to have sufficient spectral resolution within the nitroxide spectrum, but still enough excitation bandwidth to cover all dipolar frequencies, estimated from the single-position RIDME experiments with hard m.w. pulses (12/24 ns). The RIDME detection positions within the nitroxide spectrum are shown in Fig. 7(A). RIDME time traces were measured with a long $t_{1}$ delay, in order to obtain single stretched exponential background. The primary time traces were background corrected with the DeerAnalysis 2016 software, using a stretched exponential fit with varying dimension parameter $n_{\mathrm{BG}}$. The form factor traces and corresponding dipolar spectra are shown in Fig. 7(B and C). All the obtained form factor traces have nearly the same modulation depth, which was equal to the corresponding 


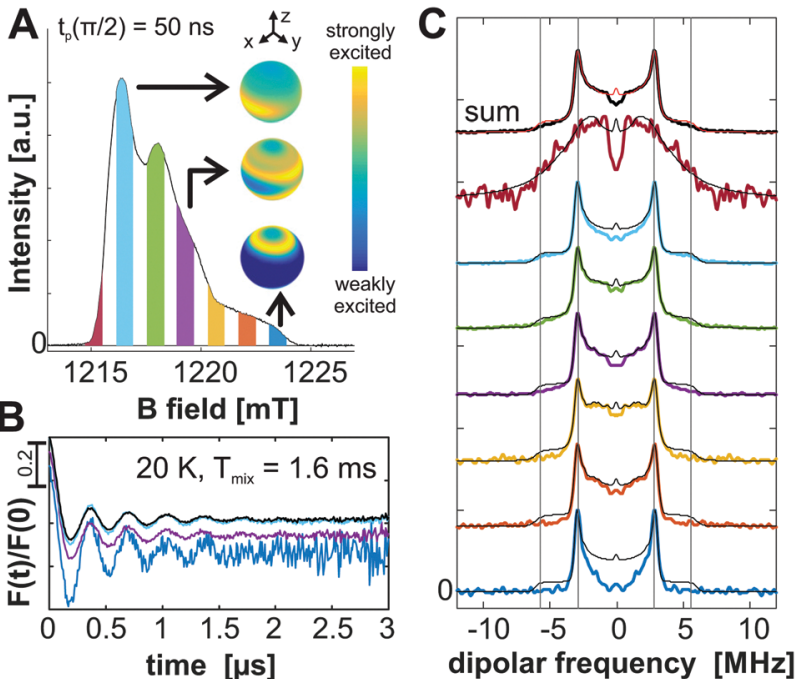

Fig. 7 Orientation selection averaging by field-stepping; (A) EDEPR of the nitroxide of $[\mathrm{Cu}(I)-\mathrm{TAHA}]$-nitroxide 1 . The shaded areas indicate which excitation bandwidth is assumed and used as a weighting factor for the individual traces. For three positions we show EasySpin ${ }^{78}$ simulations (orisel) that illustrate the expected spatial orientation of the excited nitroxide spins. (B) Form factors obtained at the field positions corresponding to the coloured bars in (A). The traces are shifted vertically for visibility. The modulation depth is approximately constant over the nitroxide spectral range. In black we show the weighted sum superimposed on the trace recorded at the maximum of the nitroxide spectrum (light blue). (C) Dipolar spectra at the various field positions; the singularities of the spectrum dominates at $z$-orientations of the nitroxide spin (dark blue). In black we show the Pake fit of each individual trace.

modulation depth in single-position RIDME measurements with hard m.w. pulses with the same mixing time. First, this confirms that our single-position measurements of the dipolar modulation buildup kinetics were accurate. Second, it confirms that for long mixing times we obtain nearly isotropic flip probability over the entire $\mathrm{Cu}$ (II) spectrum and accordingly achieve the full modulation depth also in the orientation-selective RIDME measurements.

In the SI1 (ESI $\dagger$ ) we summarised the theoretical description of the orientation averaging procedure, based on the existing orientation selection theory and the concept of conditional probability. While in practice simple approximate orientation averaging procedures show quite good performance, there are potential problems if the overall excitation profile of either A or B spins is not flat. For instance, summation of several PDS traces detected at different spectral points with rectangular pulses will almost for sure contain waves in the overall effective excitation profile due to the specific wavy shape of the excitation bandwidth of a rectangular microwave pulse, as well as due to the limited number of summed excitation profiles.

In the RIDME experiment, with respect to the $\mathrm{Cu}$ (II) (B spin) orientations we are in a good averaging situation, with almost perfectly flat excitation profile as is demonstrated by the same dipolar modulation depth values for different detection positions within the nitroxide spectrum. In other words, each of the orientation-resolved RIDME measurements corresponds to the full set of dipolar frequencies possible for the given set of excited nitroxide orientations, and summed over all possible $\mathrm{Cu}$ (II) orientations. A qualitative interpretation of these findings in terms of orientation of the nitroxide moiety in the [ $\mathrm{Cu}$ (II)TAHA]-nitroxide ruler 1 will be discussed in combination with the frequency swept RIDME experiments in Section 3.7.

The orientation-averaged $\mathrm{Cu}(\mathrm{II})$-nitroxide RIDME traces were obtained from the orientation-resolved RIDME data measured with the rectangular pulses setup using a procedure which is similar to the one in the recently reported work of Giannoulis et $a l .{ }^{49}$ First, all primary RIDME traces were normalised to the same signal intensity at the zero time. Second, each trace was multiplied by the nitroxide EPR intensity at the corresponding spectral position. At the third step all such scaled RIDME traces were summed to obtain an orientation-averaged RIDME trace. We also tested an option of performing the background correction prior to the orientation averaging, which was then done with the form factor traces. Due to the lower SNR in the individual traces, this version of data analysis appeared somewhat less robust. Note that detection positions within the nitroxide EPR spectrum were selected such that one could neglect the overlap of the m.w. pulse bandwidths from the neighbouring detection positions. This, however, left relatively broad gaps between the detection positions, and accurate orientation averaging was possible only because the $\mathrm{Cu}$ (II)-nitroxide dipolar spectrum changes smoothly with respect to the detection position. Covering the whole nitroxide spectrum by the bandwidths of the detection pulses would be possible if more detection positions and/or broader excitation bandwidths of the m.w. pulses were used, however then some orientations of the nitroxide radicals would be counted more than others due to excitation band overlap. (See the discussion of the eqn (S5)-(S8) in the ESI $\dagger$ ) With rectangular pulses, one of these two excitation profile problems persists, unless very elaborate data combination techniques are used. Accurately orientation-averaged dipolar evolution data are thus only accessible for cases with moderate orientation selection, where the probability distribution of orientations of the nitroxide varies smoothly with changing magnetic field. Fortunately, these cases are the most abundant ones in pulse EPR practice.

\subsection{Frequency-swept $\mathrm{Cu}$ (II)-nitroxide RIDME and geometric interpretation for [Cu(II)-TAHA]-nitroxide}

Using frequency-swept (chirped) pulses allowed us to detect the entire set of nitroxide orientations in a single experiment. An example of the chirp RIDME data processing for data obtained with the $\mathrm{Cu}(\mathrm{II})$-nitroxide ruler 1 is shown in Fig. S23-S25 (ESI $\dagger$ ). As in the case of rectangular pulses, the chirp RIDME measurements were performed with long $t_{1}$, in order to ensure a single stretched exponential background decay, and long mixing time for isotropic excitation probability distribution for the $\mathrm{Cu}$ (II) spins. The down-converted echo transients for the first few points in a RIDME experiment are shown in Fig. S23(A) (ESI $\dagger$ ). Fourier transformation gives the nitroxide EPR spectrum in frequency domain. The agreement between the field-swept echodetected EPR spectrum converted to the frequency domain, and the FT-EPR spectrum is rather good (Fig. S23(B), ESI $\dagger$ ) up to a slight shift of intensity close to the central frequencies of the 

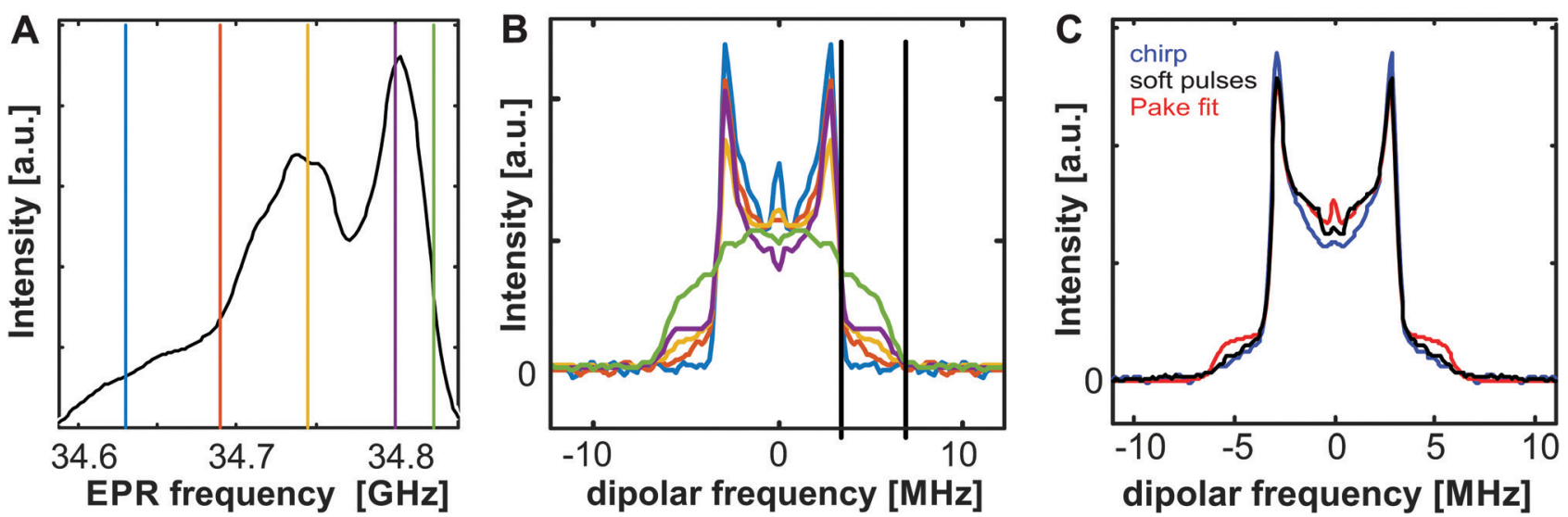

Fig. 8 Orientation selection from FT-chirped RIDME measured on [Cu(I)-TAHA]-nitroxide 1 at $20 \mathrm{~K}$ with $T_{\text {mix }}=1.6 \mathrm{~ms}$. (A) FT-EPR spectrum; the vertical coloured lines in the nitroxide spectrum indicate the positions for which the dipolar spectra are plotted in (B). (B) Dipolar spectra at several positions in the nitroxide spectrum. The singularities of a Pake pattern are predominantly found in the spectra of nitroxide spins in the $z$-orientation (blue), whereas for $x$-orientation (green) the shoulders are dominating. The black vertical lines are at $\omega_{\mathrm{dd}, \perp}(r=2.5 \mathrm{~nm})$, resp. $2 \omega_{\mathrm{dd}, \perp}$. (C) The orientationaveraged spectra from field-stepped averaging (black) and chirp excitation (blue) are compared to a Pake fit (red). Clearly in both cases the shoulders of the dipolar spectrum (here at $\approx 5 \mathrm{MHz}$ ) differ from the Pake lineshape. The slight difference between the averaged spectra of the two methods at low frequencies may be due to a residual contribution of the background fitting artifact.

spectrum. This might be due to imperfect offset compensation, and thus partial suppression of signal from destructive interference or due to the inhomogeneous detection efficiency imposed by the resonator. Resonator and pulse excitation profiles for these experiments can be found in Fig. S23(C) (ESI $\dagger$ ).

After background correction along the RIDME time dimension, and the corresponding Fourier transform, the 2D EPR-correlated dipolar coupling pattern is obtained. For selected positions in the nitroxide spectrum (Fig. 8(A)) we show the projections of the dipolar spectra in Fig. 8(B). They closely resemble the spectra obtained with field-stepped RIDME (Fig. 7(C)). The EPR-resolved dipolar coupling pattern for [Cu(II)-TAHA]-nitroxide $\mathbf{1}$ is shown in Fig. 9(A).

The orientation averaged spectra for the two averaging methods are compared to a Pake fit in Fig. 8(C). Interestingly, the two schemes produce very similar results, but the shape of the dipolar pattern does not exactly match the shape of the predicted Pake fit (red). In both cases the best fit to the 'classical' Pake pattern is observed at the maximum of the nitroxide spectrum. This deviation cannot be explained easily by incomplete excitation of orientations any more. Both averaging schemes are designed to excite all possible nitroxide orientations, and in the case of the chirped experiment this uniform excitation is demonstrated by noting that the FT-EPR spectrum up to minor differences gives the expected lineshape. Furthermore, the modulation depth is approximately constant over the whole nitroxide spectral range (compare Fig. S25(B) for chirp experiment, ESI $\dagger$ ), which is consistent with our hypothesis that at the chosen mixing time all $\mathrm{Cu}$ (II) orientations contribute with equal probability to dipolar modulation. It may still be that at the chosen mixing time, which was rather at the upper suggested limit in both experiments, the components of the $\mathrm{Cu}(\mathrm{II})$ orientations that relax faster (i.e. the $z$-orientations) are already in a range where they are suppressed due to multiple flipping events. This would be consistent with the observation that the total modulation depth is smaller than $\lambda_{\max }=0.5$. Another consideration is that in our analysis we neglect any influence that the $g$-factor has on the coupling frequency according to eqn (5). If, indeed, $g_{\mathrm{B}}=g_{\mathrm{Cu}}$ is strongly correlated to the observed distance one could expect a different averaged spectrum than from standard Pake powder averaging, with 'smeared out' regions of the dipolar pattern, depending on the exact geometry and $g$-strain in the molecule.

While in the presented case the orientation averaging worked equally well for the rectangular and chirp pulses setups, it is expected that for cases with very strong orientation selection (high-field detection, fully rigid well-defined structures of paramagnetic moieties) the approach with chirp pulses will provide better data than the one with rectangular pulses. We expect that due to the need of only a single measurement the chirp-pulse approach will become the standard for orientation-resolved dipolar spectroscopy experiments, once AWG setups with sufficient excitation and detection bandwidth are broadly available. Several methods for the simulation and interpretation of EPR correlated dipolar coupling patterns for nitroxides have been reported previously. ${ }^{68-70,73,117}$ In the scope of this work we do not perform a full fitting of the coupling patterns, but remain with a qualitative interpretation. A graphical representation of the relevant angles is given in Fig. 1(B). Two motions of the nitroxide relative to the long axis of the ruler are possible: $\theta_{\mathrm{NO}}$ characterises the tilting of the $\mathrm{N}-\mathrm{O}$ bond relative to the long axis of the ruler, and $\phi_{\mathrm{NO}}$ describes the rotation around this axis. We can use these two angles to describe the orientation of the nitroxide spins (i.e. A spins, and thus $\left.\theta_{\mathrm{A}}, \phi_{\mathrm{A}}\right)$ as used in the Theory section of ESI. $\dagger$ To analyse the nitroxide-detected RIDME coupling patterns in terms of orientation correlation it is only relevant how $B_{0}$ is oriented with respect to the selected nitroxide spin, thus to identify $P_{\mathrm{d}}\left(\omega\left(\theta_{\mathrm{A}}, \phi_{\mathrm{A}}\right)\right)$ as defined in the Theory section of ESI. $\dagger$ Given an assignment of a dipolar sub-spectrum to such a selection of orientations we can then attribute the relative intensities in the 
A

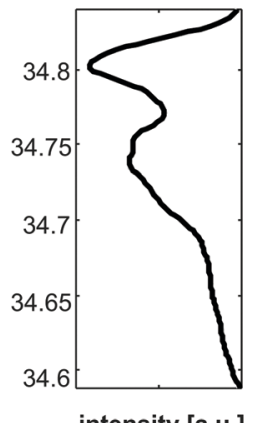

\section{-nitroxide} [Cu(II)-TAHA]
-nitroxide
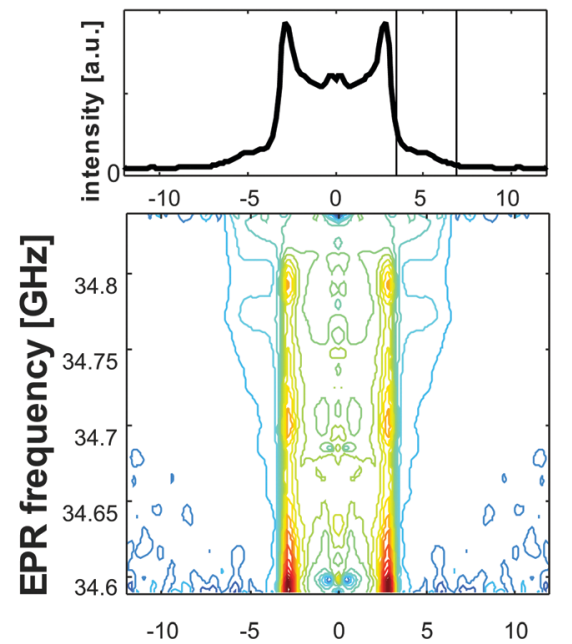

coupling frequency $[\mathrm{MHz}]$
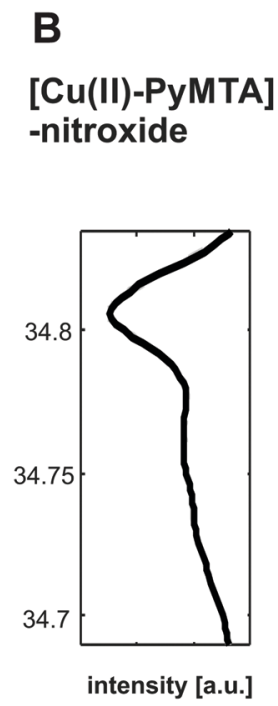
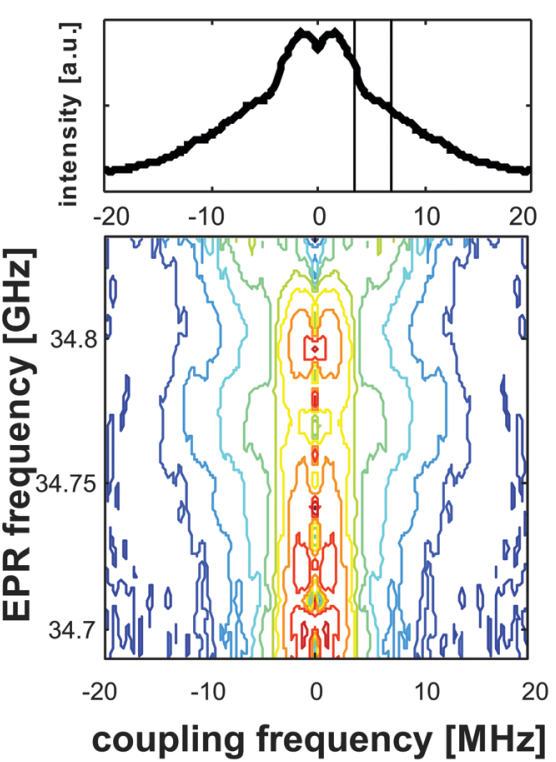

Fig. 9 2D-Fourier transform results of chirped RIDME data (A) measured on [CU(I)-TAHA]-nitroxide 1 and (B) measured on [Cu(II)-PyMTA]-nitroxide 2 at $20 \mathrm{~K}, T_{\text {mix }} 1.6 \mathrm{~ms}$ with $400 \mathrm{MHz}$ bandwidth chirped pulses, centred on nitroxide; the EPR dimension is obtained by FT of the echo transients. The resulting EPR-resolved RIDME traces are then background corrected, and the second FT yields the spectra of only the electron-electron coupling. Note that for better visibility the plot range for [Cu(II)-PyMTA]-nitroxide 2 does not cover the entire nitroxide spectrum (for more information on chirp excitation and data processing see ESI $\dagger$ ).

Pake-like pattern to preferential orientation of the inter-spin vector with respect to $B_{0}$ (which corresponds to finding $P\left(\theta \mid \theta_{\mathrm{A}}, \phi_{\mathrm{A}}\right)$ ). With [Cu(II)-TAHA]-nitroxide 1 we observe that excitation of the $x$-orientation range in the nitroxide spectrum (see inset of Fig. 2(B)) favours a dipolar spectrum with a high representation of parallel orientation of the inter-spin vector $\left(\theta=0^{\circ}\right)$, whereas excitation of $z$-orientations is linked to perpendicular orientations $\left(\theta=90^{\circ}\right)$. We see no strong differences in the dipolar coupling pattern between $y$-, and $z$-orientations of the nitroxide. This indicates cylindrical symmetry of the averaging of the second nitroxide angle $\phi_{\mathrm{NO}}$ around the long axis of the ruler (i.e. a cone-like distribution).

This is expected since the rotation around $\mathrm{C}-\mathrm{C}$ bonds in the spacer leads to a uniform distribution of the polar angle $\phi_{\mathrm{NO}}$ as described in Polyhach et al. ${ }^{69}$

\subsection{Comparison of RIDME results obtained with [Cu(II)-TAHA]-nitroxide and [Cu(II)-PyMTA]-nitroxide}

The projections of a chirp RIDME measurement on [Cu(II)-PyMTA]nitroxide 2 are shown in Fig. 11 and orientation-averaged RIDME data for the $\mathrm{Cu}(\mathrm{II})$-nitroxide rulers $\mathbf{1}$ and $\mathbf{2}$ are compared in Fig. 10. Nitroxide and Cu(II) EDEPR spectra and continuous wave X-band spectra for the two rulers are shown in Fig. $\mathrm{S} 1$ (ESI $\dagger$ ). $\mathrm{Cu}(\mathrm{II})$ in ruler 2 exhibits somewhat weaker $g$-tensor anisotropy and less resolved hyperfine coupling in the parallel orientation: four steps in the low field part of the EDEPR spectrum are visible for [Cu(II)-TAHA]-nitroxide $\mathbf{1}$, but not for [Cu(II)-PyMTA]-nitroxide 2. For the same mixing time, we obtained nearly the same dipolar modulation depths for the two rulers, which is consistent with the observation that the relaxation properties of $\mathrm{Cu}(\mathrm{II})$ in the rulers $\mathbf{1}$ and 2 are very similar (see SI1, ESI $\dagger$ ). The dipolar evolution traces reveal striking difference between the two rulers (Fig. 10). For ruler 1 several dipolar oscillations are detected, the corresponding distance distribution is narrow, and is in agreement with the expected spin-spin distance according to the molecular structure. For ruler 2, however, we observed fast dipolar signal decay without any pronounced oscillations, encoding a broad dipolar spectrum
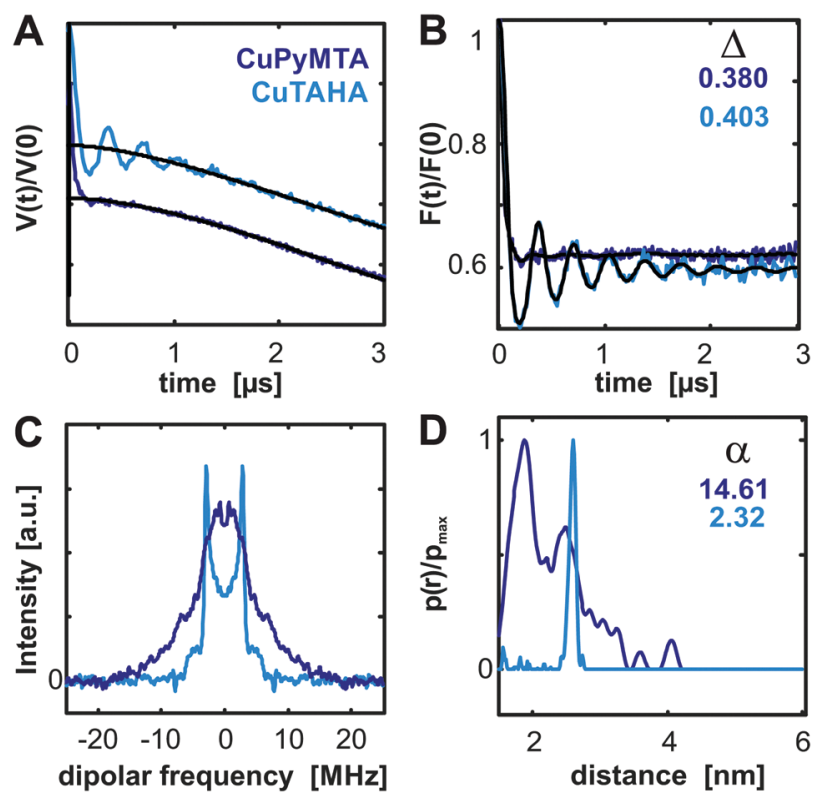

Fig. 10 Comparison of the results of RIDME experiments on the [Cu(॥)-TAHA]-nitroxide 1 (light blue, $t_{1}=4100 \mathrm{~ns}$ ) and [Cu(॥)-PyMTA]nitroxide 2 (purple, $t_{1}=6100 \mathrm{~ns}$ ) at $30 \mathrm{~K}$; (A) primary data and background fit with a stretched exponential function; while clearly defined oscillations are visible in the case of ruler $\mathbf{1}$, these cannot be visually identified in the case of ruler 2. (B) Form factors; the modulation depth $\Delta$ is similar in both samples. (C) Dipolar spectra; contributions from significantly higher frequency are observed in the latter case, which lead to intensity at short distances in the distribution. (D) Distance distributions; the regularisation parameter $\alpha$ is given in the panel. 

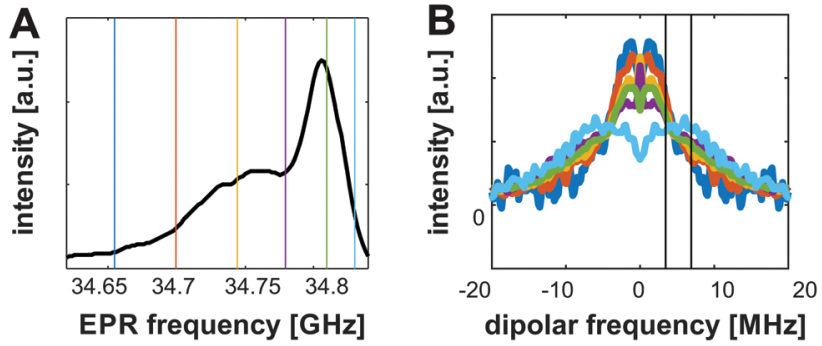

Fig. 11 (A) FT-EPR spectrum of the nitroxide of ruler 2; (B) dipolar spectra $a$ the spectral positions indicated in (A) measured with the chirped RIDME experiment. The chirped RIDME over the full nitroxide range of $[\mathrm{Cu}(I)-$ PyMTA]-nitroxide 2 was measured with long $t_{1}=3500$ ns. The dipolar spectra are much broader than those of $[\mathrm{Cu}(I)-\mathrm{TAHA}]$-nitroxide 1 , which most likely arises from exchange coupling. The black vertical lines are at $\omega_{\mathrm{dd}, \perp}(r=2.5 \mathrm{~nm})$, resp. $2 \omega_{\mathrm{dd}, \perp}$

and a too broad apparent spin-spin distance distribution, far beyond the expected flexibility of the ruler. This suggests that the two different ligands lead to a different transfer of spin density into the $\pi$-system of the spacer that connects the two spin labels and thus to different exchange coupling between $\mathrm{Cu}$ (II) and nitroxide. Note that the two rulers differ in length by only about one $\mathrm{C}-\mathrm{C}$ bond. However, in the case of TAHA as the ligand (ruler $\mathbf{1}$ ) all $\mathrm{N}$ atoms are separated from the $\pi$-conjugated spacer by three saturated bonds, whereas in case of PyMTA as the ligand (ruler 2) two $\mathrm{N}$ atoms are separated by only two saturated bonds and, most importantly, one $\mathrm{N}$ atom is an integral part of the $\pi$-system of the spacer. The spacer is in conjugation with the two olefinic $\mathrm{C}$ atoms of the nitroxide moiety where some of the spin density of the nitroxide is located. Therefore, exchange coupling in ruler 2 is the most plausible reason of the observed unexpectedly broad distribution of the dipolar frequencies. Note that presence of a major fraction of ruler molecules with strong exchange couplings, not detectable by the RIDME experiment, can be immediately excluded by noticing no changes in the EPR line shapes of $\mathrm{Cu}$ (II) and nitroxide moieties, as well as no significant loss in the dipolar modulation depths as compared to the anticipated values. We are currently investigating this phenomenon in detail, involving quantum chemical calculations of the spin density distributions in such compounds, comparison of the dipolar spectra in the $\mathrm{Cu}$ (II)-nitroxide and corresponding $\mathrm{Cu}$ (II)-Cu(II) compounds, as well as fitting the exchange coupling distributions. The order of magnitude of the exchange coupling is readily estimated to be similar to the width of the sum projection on top of the $2 \mathrm{D}$ contour plot in Fig. 9B. A detailed analysis will be published elsewhere. Besides the complication by the exchange coupling, the overall performance of the chirp RIDME measurements with compound 2 is good and comparable to the chirp RIDME on compound 1.

\subsection{Comparison of Cu(II)-nitroxide DEER and RIDME measurements at Q-band}

We compared RIDME and DEER measurements on the [Cu(II)TAHA]-nitroxide ruler $\mathbf{1}$, for which the dipole-dipole interaction is dominant and the shape of the dipolar spectrum has clear features of the canonical Pake pattern. For this ruler the separation between EPR absorption maxima of the nitroxide spins and the $\mathrm{Cu}$ (II) ions is $\Delta B=33 \mathrm{mT}$, which approximately translates to the resonance frequency difference $\Delta \nu \approx 920 \mathrm{MHz}$ (see Fig. 2(A)). Even with the recent major improvements in EPRresonator technology ${ }^{66}$ and UWB excitation ${ }^{63,64}$ this large resonance frequency offset remains challenging for DEER spectroscopy. It is not a unique case: maximum absorption in the $\mathrm{Cu}$ (II) EPR spectrum is observed at a similar field for [Cu(II)-PyMTA]-nitroxide 2 as well as for $[\mathrm{Cu}(\mathrm{II})$-terpyridine $]$-nitroxide. ${ }^{28}$ At $\mathrm{X}$-band frequencies, DEER measurements between the maxima of the nitroxide and $\mathrm{Cu}(\mathrm{II})$ spectra are facile with commercial resonators and hardware, but orientation averaging is challenging. ${ }^{17}$

In order to qualitatively compare the performance of the DEER and RIDME experiments in this case, we conducted the following two measurements. Both experiments were set up in the same spectrometer session on a $200 \mu \mathrm{M}$ sample of [Cu(II)-TAHA]-nitroxide 1, see Fig. 12. First, we set up a DEER experiment with the shortest achievable 48/96 ns pulses for detection on the maximum of the $\mathrm{Cu}$ (II) spectrum. For pumping the nitroxide spins we used a chirp pulse of duration $t_{\mathrm{p}}=150 \mathrm{~ns}$ and of bandwidth $450 \mathrm{MHz}$. To have a pump pulse that is
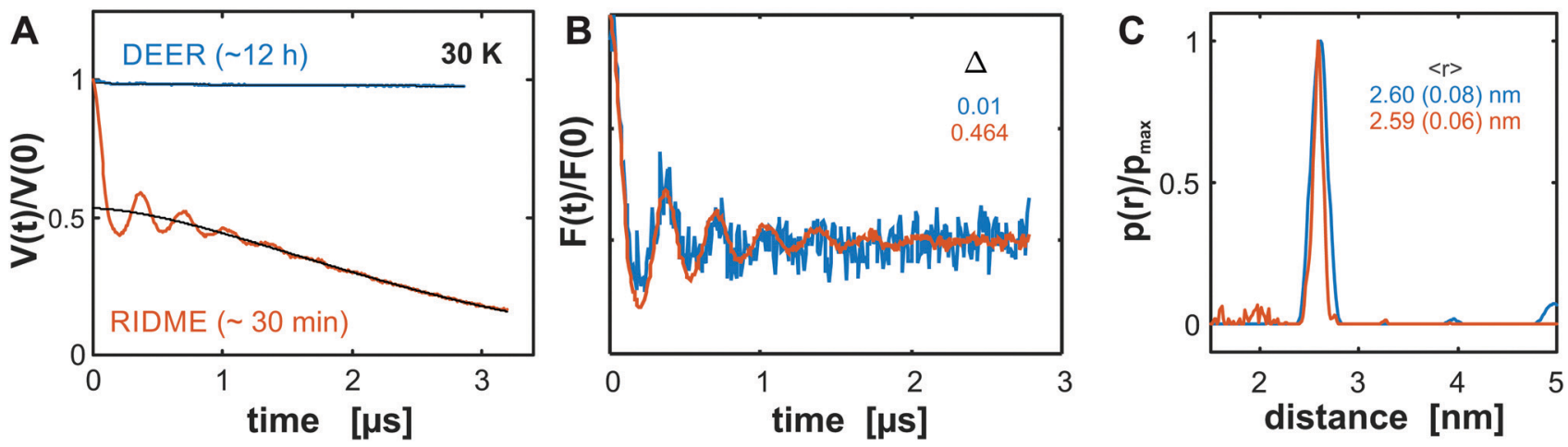

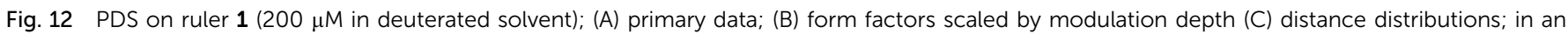

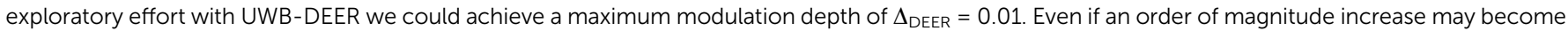

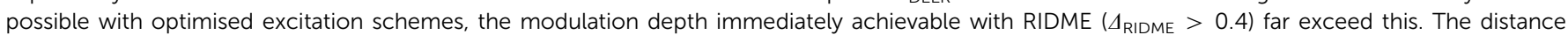
distributions obtained with the two methods are in excellent agreement. 
approximately centred in the nitroxide EPR spectrum we used an offset of pump and detection pulse of $+900 \mathrm{MHz}$. The refocusing delays were set to $t_{1}=400 \mathrm{~ns}$ and $t_{2}=3500 \mathrm{~ns}$. Due to the strong resonator over-coupling, the critical adiabaticity of the chirp pulse was small, and the experimentally observed inversion efficiency was only 1\% (Fig. 12). Note that higher resonator bandwidth and better power conversion could have been achieved with a loop-gap resonator for $1.6 \mathrm{~mm}$ samples, ${ }^{66}$ albeit at the expense of a reduction of the echo amplitude by about a factor of two.

Second, we performed a RIDME experiment in the following way. The resonator mode was changed to a narrow profile (as seen in the tune picture). The detection frequency was set in the centre of the resonator, to have maximum sensitivity. With such a setup, the detection $(\pi / 2) /(\pi)$ pulses of the length of $12 / 24$ ns could be used. The magnetic field was set to the maximum of the nitroxide EPR spectrum at the detection frequency. The transverse evolution delays were set to $t_{1}=t_{2}=$ $3500 \mathrm{~ns}$, in order to avoid the background shape problem, which at the same time removed the ESEEM artefacts from the RIDME trace. The mixing time of $T_{\text {mix }}=400 \mu \mathrm{s}$ was used.

The time-increment for shifting the position of both pump pulse (in DEER) and the mixing block (in RIDME) was set to $8 \mathrm{~ns}$. In the DEER experiment, a reduced number of data points was detected, so that not the full range of available transverse evolution period was measured and thus a small additional increase in SNR could be achieved.

The detected RIDME modulation depth was $46 \%$ against $1 \%$ in DEER, which ensured a major SNR advantage for RIDME in the comparison of the form factor traces. Both measurements revealed a sharp peak at $2.6 \mathrm{~nm}$ (2.59 $\mathrm{nm}$ for DEER), which was slightly broader in the DEER case (FWHM of $0.08 \mathrm{~nm}$ ) as compared to the RIDME data (FWHM of $0.06 \mathrm{~nm}$ ). The small differences in position and width of the distance peak from the DEER data are likely due to the higher noise level in those data, but could be as well related to a stronger orientation selection effect in this measurement. Furthermore, a smearing of the DEER time trace, due to the $150 \mathrm{~ns}$ long pump pulse is expected, which would also affect the shape of the distance distribution. ${ }^{61}$

Although a DEER experiment with a dedicated ultra-wideband resonator for $1.6 \mathrm{~mm}$ sample tube and systematic chirp pulse optimisation is expected to perform better, the comparison is still representative for a large resonance offset between the pumped and detected species. The RIDME experiment outperforms DEER in this regime. Only the use of bimodal resonators with tunable frequency offset between the two modes could be expected to change this. Thus, for spin pairs consisting of an organic radical and a metal ion complex with a spectral offset from the $g=g_{\text {e }}$ condition by approximately $1 \mathrm{GHz}$ or even more, the RIDME experiment is an advantageous and robust alternative to DEER. Nevertheless there is a clear motivation for the further development of UWB-DEER on such systems as [Cu(II)-TAHA]-nitroxide 1, because the detection on the $\mathrm{Cu}$ (II) spins may reveal the orientation of the $\mathrm{Cu}$ (II) complex with respect to the dipolar axis. This is not possible from nitroxide detected RIDME, where we have shown that the orientations of the $\mathrm{Cu}$ (II) spins are averaged when using optimal mixing times. If we assume, however, that the frequency swept inversion pulse in the UWB DEER experiment has uniformly inverted the nitroxide spins, we have in this dataset the complementary information to the RIDME experiment. Then we can again assume orientation averaging of the B spins (this time the nitroxide spins) and analyse the dipolar spectrum in terms of the orientation of the detected $\mathrm{Cu}$ (II) spins. In our particular case the SNR of the DEER traces does not allow a detailed interpretation, but it appears that the shoulders of the Pake-like pattern are slightly suppressed. The detection on the maximum of the $\mathrm{Cu}$ (II) spectrum primarily excites spins with $x / y$-orientation, which are not resolved in cases like this with nearly perfect axial symmetry (compare spectra of the Cu(II)-TAHA complex in the Fig. S1, ESI $\dagger$ ).

This suggests that the Cu(II) complex is oriented in such a way that the $g_{z}$ component is aligned with the dipolar axis as is expected based on the molecular structure of the ruler. As in the case of the nitroxide, the free rotation around $\mathrm{C}-\mathrm{C}$ single bonds in the spacer implies ensemble averaging of the $x$ and $y$ component orientation relative to the spacer moiety. Identifying this orientation of the $g$-tensor with the unique axis in the axially symmetric $\mathrm{Cu}(\mathrm{II})-\mathrm{TAHA}$ complex allows a tentative prediction of the relative alignment of the nitroxide and the $\mathrm{Cu}$ (II) in [Cu(II)-TAHA]-nitroxide 1: the spins are preferentially aligned such that the $x$-axis of the $g$-tensor of the nitroxide, resp. the $z$-axis of the $g$-tensor of the $\mathrm{Cu}$ (II) spin (in their respective principle axis systems) point approximately along the ruler backbone axis. Note that we attempted to confirm this interpretation by measuring chirped RIDME with a very short mixing time (data not shown). When using $T_{\text {mix }}$ that is on the timescale of $T_{1, \mathrm{Cu}}$ or even shorter we expect to suppress the $x / y$-orientations of the $\mathrm{Cu}$ (II) spins, which we have observed to feature slightly slower longitudinal relaxation, relative to the $z$-orientations (Fig. S7, ESI $\dagger$ ). Unfortunately, but predictably, the total modulation depth, and thus the sensitivity, in such measurements is significantly lower throughout the nitroxide spectral dimension, and we observed much higher artifact levels form crossing echos and ESEEM, which prevented detailed interpretation of the data. This may be different for systems with a more pronounced differential relaxation of the orientations of the $\mathrm{Cu}(\mathrm{II})$ spins.

\section{Conclusion}

In this work, we focused on the accuracy of different steps in the measurement and data processing of nitroxide detected $\mathrm{Cu}(\mathrm{II})-$ nitroxide RIDME. While some phenomena in $\mathrm{Cu}(\mathrm{II})$-nitroxide RIDME, in particular regarding the background decay shape, are not yet fully understood, the measurements and their analysis can be done in a reproducible and robust way. At the expense of some sensitivity loss, the background problem can be circumvented by using a sufficiently long first interpulse delay in the observer sequence. Importantly, this modification of the RIDME method will allow for more accurate distance measurements in the cases of broad distance distributions, where the presence of the background artefact is a serious problem.

We also further elaborated on the issue of the ESEEM artefact suppression, and proposed an improved approach, based on the 
elongation of the first inter-pulse delay, complemented with the previously reported ESEEM artefact averaging over delay time variations that now also maintains the constant-time property of the basic RIDME experiment.

In this study we used the water soluble rulers [Cu(II)-TAHA]nitroxide 1 and [Cu(II)-PyMTA]-nitroxide 2 . Their syntheses and with this a general access to water soluble rulers of the type [metal ion-ligand]-nitroxide were presented. The two rulers 1 and 2 differ essentially only in the type of $\mathrm{Cu}(\mathrm{II})$ coordinating ligand. The similarity of the relaxation properties of the three complexes $\mathrm{Cu}$ (II)-TAHA, Cu(II)-PyMTA, and Cu(II)-terpyridine ${ }^{88}$ and their comparable performance in RIDME suggests that $\mathrm{Cu}$ (II) complexes are a rather robust family of spin labels with no need for additional elaborate optimisation of RIDME experimental protocols for a particular complex. We recommend a measurement temperature between $20 \mathrm{~K}$ and $30 \mathrm{~K}$, with lower temperatures being favourable for long distances, and higher temperatures for short distances. Direct coordination of $\mathrm{Cu}$ (II) by an atom being part of the $\pi$-system in the spacer leads to exchange interaction of the same order of magnitude as the dipole-dipole interaction at distances around $2.5 \mathrm{~nm}$. This problem should not be encountered in spin labelling studies with proteins, where extended $\pi$-conjugation is usually not expected. It should, however, be taken into consideration when planning the attachment of such a spin label to a compound with a $\pi$-system, e.g. nucleic acids or light harvesting complexes. Because in $\mathrm{Cu}$ (II)-TAHA all donor atoms of the ligand are essentially electronically decoupled from the atom through which the label is attached to the molecule of interest, this complex appears as a valuable $\mathrm{Cu}(\mathrm{II})$-based spin label.

RIDME experiments simplify orientation averaging for strongly anisotropic spin Hamiltonians considerably, since in the mixing time limit of maximum modulation depth orientation selection is negligible for the inverted spins. Hence, the optimal averaging scheme becomes independent of system geometry and requires only uniform detection of the observer spin. For [Cu(II)-TAHA]-nitroxide we could achieve this by a series of RIDME measurements with soft microwave pulses as well as by complete excitation of the nitroxide spectrum with a chirp observer sequence. The latter approach should be applicable even for stronger orientation correlation.

We found that with typically available microwave resonators, RIDME outperforms DEER for Cu(II)-nitroxide systems in terms of sensitivity by a large margin, even if broadband chirp pump pulses are used in DEER. The relatively poor DEER performance results from the large frequency difference between the maxima of both spectra and is thus also expected for several other combinations of metal ion complexes with organic radicals, in particular at Q-band and higher frequencies.

To summarize, we believe that the RIDME technique is wellsuited for detecting dipolar interactions in $\mathrm{Cu}$ (II)-nitroxide spin pairs and that it has been developed to a point where practical applications in materials science and structural biology are reliable.

\section{Conflicts of interest}

There are no conflicts of interest to declare.

\section{Acknowledgements}

This work was supported by the Deutsche Forschungsgemeinschaft (DFG) within SPP 1601 (GO555/6-2) and by SNF grant 200020 157034. We acknowledge one of the reviewer's of this manuscript for the suggestion to check if the division approach is also capable of removing the RIDME background artefact.

\section{Notes and references}

1 T. Prisner, M. Rohrer and A. F. MacMillan, Annu. Rev. Phys. Chem., 2001, 52, 279-313.

2 G. Jeschke and Y. Polyhach, Phys. Chem. Chem. Phys., 2007, 9, 1895-1910.

3 G. Jeschke, Annu. Rev. Phys. Chem., 2012, 63, 419-446.

4 E. Bordignon, EPR Spectroscopy, Springer, Berlin, Heidelberg, 2011, pp. 121-157.

5 P. P. Borbat and J. H. Freed, Structural Information from Spin-Labels and Intrinsic Paramagnetic Centres in the Biosciences, Springer, Berlin, Heidelberg, 2013, pp. 1-82.

6 W. L. Hubbell, C. J. López, C. Altenbach and Z. Yang, Curr. Opin. Struct. Biol., 2013, 23, 725-733.

7 A. Caneschi, D. Gatteschi, R. Sessoli and P. Rey, Acc. Chem. Res., 1989, 22, 392-398.

8 C. Benelli and D. Gatteschi, Chem. Rev., 2002, 102, 2369-2388.

9 D. Gatteschi, A.-L. Barra, A. Caneschi, A. Cornia, R. Sessoli and L. Sorace, Coord. Chem. Rev., 2006, 250, 1514-1529.

10 S. L. Veber, M. V. Fedin, A. I. Potapov, K. Y. Maryunina, G. V. Romanenko, R. Z. Sagdeev, V. I. Ovcharenko, D. Goldfarb and E. G. Bagryanskaya, J. Am. Chem. Soc., 2008, 130, 2444-2445.

11 M. Fedin, S. Veber, I. Gromov, K. Maryunina, S. Fokin, G. Romanenko, R. Sagdeev, V. Ovcharenko and E. Bagryanskaya, Inorg. Chem., 2007, 46, 11405-11415.

12 M. Fedin, V. Ovcharenko, R. Sagdeev, E. Reijerse, W. Lubitz and E. Bagryanskaya, Angew. Chem., Int. Ed., 2008, 47, 6897-6899.

13 M. V. Fedin, S. L. Veber, K. Y. Maryunina, G. V. Romanenko, E. A. Suturina, N. P. Gritsan, R. Z. Sagdeev, V. I. Ovcharenko and E. G. Bagryanskaya, J. Am. Chem. Soc., 2010, 132, 13886-13891.

14 J. van Slageren, Top. Curr. Chem., 2012, 321, 199-234.

15 S. Richert, J. Cremers, H. L. Anderson and C. R. Timmel, Chem. Sci., 2016, 7, 6952-6960.

16 S. Richert, J. Cremers, I. Kuprov, M. D. Peeks, H. L. Anderson and C. R. Timmel, Nat. Commun., 2017, 8, 14842.

17 N. Evelyn, G. Adelheid and J. Gunnar, Angew. Chem., Int. Ed., 2002, 41, 3907-3910.

18 B. E. Bode, J. Plackmeyer, T. F. Prisner and O. Schiemann, J. Phys. Chem. A, 2008, 112, 5064-5073.

19 B. E. Bode, J. Plackmeyer, M. Bolte, T. F. Prisner and O. Schiemann, J. Organomet. Chem., 2009, 694, 1172-1179.

20 Z. Yang, D. Kise and S. Saxena, J. Phys. Chem. B, 2010, 114, 6165-6174.

21 J. Sarver, K. I. Silva and S. Saxena, Appl. Magn. Reson., 2013, 44, 583-594. 
22 Z. Yang, G. Jimnez-Oses, C. J. Lopez, M. D. Bridges, K. N. Houk and W. L. Hubbell, J. Am. Chem. Soc., 2014, 136, 15356-15365.

23 I. M. C. van Amsterdam, M. Ubbink, G. W. Canters and M. Huber, Angew. Chem., Int. Ed., 2003, 42, 62-64.

24 J. S. Becker and S. Saxena, Chem. Phys. Lett., 2005, 414, 248-252.

25 S. Richert, I. Kuprov, M. D. Peeks, E. A. Suturina, J. Cremers, H. L. Anderson and C. R. Timmel, Phys. Chem. Chem. Phys., 2017, 19, 16057-16061.

26 M. Ji, S. Ruthstein and S. Saxena, Acc. Chem. Res., 2014, 47, 688-695.

27 Z. Yang, M. Ji, T. F. Cunningham and S. Saxena, Methods in Enzymology, Academic Press, 2015, vol. 563, pp. 459-481.

28 A. Meyer, D. Abdullin, G. Schnakenburg and O. Schiemann, Phys. Chem. Chem. Phys., 2016, 18, 9262-9271.

29 A. Giannoulis, M. Oranges and B. E. Bode, ChemPhysChem, 2017, 18, 2318-2321.

30 R. Meng, J. Becker, F. T. Lin, S. Saxena and S. G. Weber, Inorg. Chim. Acta, 2005, 358, 2933-2942.

31 J. S. Becker and S. Saxena, Chem. Phys. Lett., 2005, 414, 248-252.

32 C. W. M. Kay, H. El Mkami, R. Cammack and R. W. Evans, J. Am. Chem. Soc., 2007, 129, 4868-4869.

33 B.-K. Shin and S. Saxena, Biochemistry, 2008, 47, 9117-9123.

34 K. J. Waldron, J. C. Rutherford, D. Ford and N. J. Robinson, Metalloproteins and metal sensing, 2009.

35 Z. Yang, M. Ji and S. Saxena, Appl. Magn. Reson., 2010, 39, 487-500.

36 B.-K. Shin and S. Saxena, J. Phys. Chem. B, 2011, 115, 15067-15078.

37 A. V. Astashkin, Methods in Enzymology, Academic Press, 2015, vol. 563, pp. 251-284.

38 M. J. Lawless, J. L. Sarver and S. Saxena, Angew. Chem., 2017, 129, 2147-2149.

39 A. Lewin, J. P. Hill, R. Boetzel, T. Georgiou, R. James, C. Kleanthous and G. R. Moore, Inorg. Chim. Acta, 2002, 331, 123-130.

40 T. F. Cunningham, M. D. Shannon, M. R. Putterman, R. J. Arachchige, I. Sengupta, M. Gao, C. P. Jaroniec and S. Saxena, J. Phys. Chem. B, 2015, 119, 2839-2843.

41 M. Yulikov, Electron Paramagnetic Resonance: Volume 24, The Royal Society of Chemistry, 2015, vol. 24, pp. 1-31.

42 L. Garbuio, E. Bordignon, E. K. Brooks, W. L. Hubbell, G. Jeschke and M. Yulikov, J. Phys. Chem. B, 2013, 117, 3145-3153.

43 S. Razzaghi, E. K. Brooks, E. Bordignon, W. L. Hubbell, M. Yulikov and G. Jeschke, ChemBioChem, 2013, 14, 1883-1890.

44 B. Joseph, V. M. Tormyshev, O. Y. Rogozhnikova, D. Akhmetzyanov, E. G. Bagryanskaya and T. F. Prisner, Angew. Chem., Int. Ed., 2016, 55, 11538-11542.

45 S. Kucher, S. Korneev, S. Tyagi, R. Apfelbaum, D. Grohmann, E. A. Lemke, J. P. Klare, H.-J. Steinhoff and D. Klose, J. Magn. Reson., 2017, 275, 38-45.

46 Z. Wu, A. Feintuch, A. Collauto, L. A. Adams, L. Aurelio, B. Graham, G. Otting and D. Goldfarb, J. Phys. Chem. Lett., 2017, 8, 5277-5282.
47 C. Gmeiner, G. Dorn, F. H. T. Allain, G. Jeschke and M. Yulikov, Phys. Chem. Chem. Phys., 2017, 19, 28360-28380.

48 C. Gmeiner, D. Klose, E. Mileo, V. Belle, S. R. A. Marque, G. Dorn, F. H. T. Allain, B. Guigliarelli, G. Jeschke and M. Yulikov, J. Phys. Chem. Lett., 2017, 8, 4852-4857.

49 A. Giannoulis, C. L. Motion, M. Oranges, M. Bühl, G. M. Smith and B. E. Bode, Phys. Chem. Chem. Phys., 2018, 20, 2151-2154.

50 The synthesis of the parent complex $\left(\mathrm{H}^{+}\right)_{x}\left(\mathrm{Na}^{+}\right)_{4-x}[\mathrm{Cu}(\mathrm{II})-$ TAHA $]^{4-}$ (20) (see SI2, ESI $\dagger$ ) started from $\mathrm{H}_{6}$ TAHA. $n \mathrm{CF}_{3} \mathrm{CO}_{2} \mathrm{H}$ (26) with a known content of the structural motive $\mathrm{H}_{6}$ TAHA. After addition of $\mathrm{CuCl}_{2}$ the $\mathrm{pH}$ was adjusted to $\mathrm{pH} 7$ through adding aqueous NaOD. Knowing the molar amount of added $\mathrm{NaOD}$ an average protonation degree of 1.9 protons per molecule was calculated. Please note, that several species differing in the protonation degree will be present. Calculation of the protonation degree of the ligand itself, $\mathrm{H}_{6}$ TAHA, with the reported $\mathrm{p} K_{\mathrm{a}}$ values (ref. 81) gave on average 1.7 protons per molecule at $\mathrm{pH} 7$ which corresponds to a mixture consisting mainly of $\mathrm{H}_{2} \mathrm{TAHA}^{4-}$ and HTAHA ${ }^{5-}$ (on average $\mathrm{H}_{1.7} \mathrm{TAHA}^{4.3-}$ ). We assume that the same applies to ruler $\mathbf{1}$.

51 W. W. Xie and P. R. Tremaine, J. Solution Chem., 1999, 28, 291-325.

52 P. P. Borbat and J. H. Freed, Chem. Phys. Lett., 1999, 313, 145-154.

53 G. Jeschke, M. Pannier, A. Godt and H. W. Spiess, Chem. Phys. Lett., 2000, 331, 243-252.

54 P. Schöps, P. E. Spindler, A. Marko and T. F. Prisner, J. Magn. Reson., 2015, 250, 55-62.

55 A. Doll and G. Jeschke, Phys. Chem. Chem. Phys., 2016, 18, 23111-23120.

56 A. D. Milov, A. G. Maryasov and Y. D. Tsvetkov, Appl. Magn. Reson., 1998, 15, 107-143.

57 V. V. Kurshev, A. M. Raitsimring and Y. D. Tsvetkov, J. Magn. Reson., 1989, 81, 441-454.

58 L. V. Kulik, S. A. Dzuba, I. A. Grigoryev and Y. D. Tsvetkov, Chem. Phys. Lett., 2001, 343, 315-324.

59 S. Milikisyants, F. Scarpelli, M. G. Finiguerra, M. Ubbink and M. Huber, J. Magn. Reson., 2009, 201, 48-56.

60 P. E. Spindler, Y. Zhang, B. Endeward, N. Gershernzon, T. E. Skinner, S. J. Glaser and T. F. Prisner, J. Magn. Reson., 2012, 218, 49-58.

61 A. Doll, S. Pribitzer, R. Tschaggelar and G. Jeschke, J. Magn. Reson., 2013, 230, 27-39.

62 C. E. Tait and S. Stoll, Phys. Chem. Chem. Phys., 2016, 18, 18470-18485.

63 A. Doll and G. Jeschke, J. Magn. Reson., 2017, 280, 46-62.

64 P. E. Spindler, P. Schöps, A. M. Bowen, B. Endeward and T. F. Prisner, eMagRes, 2016.

65 A. Doll and G. Jeschke, J. Magn. Reson., 2014, 246, 18-26.

66 R. Tschaggelar, F. D. Breitgoff, O. Oberhänsli, M. Qi, A. Godt and G. Jeschke, Appl. Magn. Reson., 2017, 48, 1273-1300.

67 A. M. Bowen, C. E. Tait, C. R. Timmel and J. R. Harmer, Structural Information from Spin-Labels and Intrinsic Paramagnetic Centres in the Biosciences, Springer, Berlin, Heidelberg, 2013, pp. 283-327. 
68 V. P. Denysenkov, T. F. Prisner, J. Stubbe and M. Bennati, Proc. Natl. Acad. Sci. U. S. A., 2006, 103, 13386-13390.

69 Y. Polyhach, A. Godt, C. Bauer and G. Jeschke, J. Magn. Reson., 2007, 185, 118-129.

70 A. Marko, D. Margraf, P. Cekan, S. T. Sigurdsson, O. Schiemann and T. F. Prisner, Phys. Rev. E: Stat., Nonlinear, Soft Matter Phys., 2010, 81, 021911.

71 V. P. Denysenkov, T. F. Prisner, J. Stubbe and M. Bennati, Proc. Natl. Acad. Sci. U. S. A., 2006, 103, 13386-13390.

72 B. Endeward, J. A. Butterwick, R. MacKinnon and T. F. Prisner, J. Am. Chem. Soc., 2009, 131, 15246-15250.

73 O. Schiemann, P. Cekan, D. Margraf, T. F. Prisner and S. T. Sigurdsson, Angew. Chem., Int. Ed., 2009, 48, 3292-3295.

74 A. Marko, D. Margraf, H. Yu, Y. Mu, G. Stock and T. Prisner, J. Chem. Phys., 2009, 130, 064102.

75 C. Abé, D. Klose, F. Dietrich, W. H. Ziegler, Y. Polyhach, G. Jeschke and H.-J. Steinhoff, J. Magn. Reson., 2012, 216, 53-61.

76 K. Keller, V. Mertens, M. Qi, A. I. Nalepa, A. Godt, A. Savitsky, G. Jeschke and M. Yulikov, Phys. Chem. Chem. Phys., 2017, 19, 17856-17876.

77 K. Keller, A. Doll, M. Qi, A. Godt, G. Jeschke and M. Yulikov, J. Magn. Reson., 2016, 272, 108-113.

78 S. Stoll and A. Schweiger, J. Magn. Reson., 2006, 178, 42-55.

79 R. Tschaggelar, B. Kasumaj, M. G. Santangelo, J. Forrer, P. Leger, H. Dube, F. Diederich, J. Harmer, R. Schuhmann, I. García-Rubio and G. Jeschke, J. Magn. Reson., 2009, 200, 81-87.

80 Y. Polyhach, E. Bordignon, R. Tschaggelar, S. Gandra, A. Godt and G. Jeschke, Phys. Chem. Chem. Phys., 2012, 14, 10762-10773.

81 G. Jeschke, S. Pribitzer and A. Doll, J. Phys. Chem. B, 2015, 119, 13570-13582.

82 J. M. Bohlen and G. Bodenhausen, J. Magn. Reson., Ser. A, 1993, 102, 293-301.

83 R. Viguier, G. Serratrice, A. Dupraz and C. Dupuy, Eur. J. Inorg. Chem., 2001, 1789-1795.

84 P. Lueders, G. Jeschke and M. Yulikov, J. Phys. Chem. Lett., 2011, 2, 604-609.

85 H. Jäger, A. Koch, V. Maus, H. Spiess and G. Jeschke, J. Magn. Reson., 2008, 194, 254-263.

86 D. Abdullin, F. Duthie, A. Meyer, E. S. Muller, G. Hagelueken and O. Schiemann, J. Phys. Chem. B, 2015, 119, 13534-13542.

87 A. Giannoulis, K. Ackermann, P. E. Spindler, C. Higgins, D. B. Cordes, A. M. Z. Slawin, T. F. Prisner and B. E. Bode, Phys. Chem. Chem. Phys., 2018, 20, 11196-11205.

88 A. Meyer and O. Schiemann, J. Phys. Chem. A, 2016, 120, 3463-3472.

89 P. Lueders, H. Jäger, M. A. Hemminga, G. Jeschke and M. Yulikov, J. Phys. Chem. Lett., 2012, 3, 1336-1340.

90 D. J. Hirsh, J. McCracken, R. Biczo and K.-A. Gesuelli, J. Phys. Chem. B, 2013, 117, 11960-11977.

91 I. Kaminker, H. Yagi, T. Huber, A. Feintuch, G. Otting and D. Goldfarb, Phys. Chem. Chem. Phys., 2012, 14, 4355-4358.

92 K. Keller, M. Zalibera, M. Qi, V. Koch, J. Wegner, H. Hintz, A. Godt, G. Jeschke, A. Savitsky and M. Yulikov, Phys. Chem. Chem. Phys., 2016, 25120-25135.
93 J. A. Clayton, M. Qi, A. Godt, D. Goldfarb, S. Han and M. S. Sherwin, Phys. Chem. Chem. Phys., 2017, 19, 5127-5136.

94 J. A. Clayton, K. Keller, M. Qi, J. Wegner, V. Koch, H. Hintz, A. Godt, S. Han, G. Jeschke, M. S. Sherwin and M. Yulikov, Phys. Chem. Chem. Phys., 2018, 20, 10470-10492.

95 Z. Yang, D. Kise and S. Saxena, J. Phys. Chem. B, 2010, 114, 6165-6174.

96 Z. Yang, G. Jimnez-Oses, C. J. Lopez, M. D. Bridges, K. N. Houk and W. L. Hubbell, J. Am. Chem. Soc., 2014, 136, 15356-15365.

97 J. Voss, W. L. Hubbell and H. R. Kaback, Biochemistry, 1998, 37, 211-216.

98 G. Jeschke, M. Sajid, M. Schulte, N. Ramezanian, A. Volkov, H. Zimmermann and A. Godt, J. Am. Chem. Soc., 2010, 132, 10107-10117.

99 M. Qi, M. Hülsmann and A. Godt, J. Org. Chem., 2016, 81, 2549-2571.

100 D. Sahoo, S. Thiele, M. Schulte, N. Ramezanian and A. Godt, Beilstein J. Org. Chem., 2010, 6, 57.

101 K. Hideg, L. Lex, H. O. Hankovszky and J. Tigyi, Synth. Commun., 1979, 9, 782-788. In this reference an amide formation is described through first treating the carboxylic acid with thionyl chloride in the presence of a large amount of pyridine and then adding the amine. Although the authors write of a freshly prepared acid chloride that was used for the reaction with the amine, we assume that, as in our case, an $N$-acylpyridinium salt had been formed which reacted with the amine.

102 P. Demay-Drouhard, H. Y. V. Ching, D. Akhmetzyanov, R. Guillot, L. C. Tabares, H. C. Bertrand and C. Policar, ChemPhysChem, 2016, 17, 2066-2078.

103 H. Takalo, P. Pasanen and J. Kankare, Acta Chem. Scand., Ser. B, 1988, 42, 373-377.

104 M. Sajid, G. Jeschke, M. Wiebcke and A. Godt, Chem. - Eur. J., 2009, 15, 12960-12962.

105 M. Qi, M. Hülsmann and A. Godt, Synthesis, 2016, 3773-3784. 106 D. Wang, M. Zhao, X. Liu, Y. Chen, N. Li and B. Chen, Org. Biomol. Chem., 2011, 10, 229-231.

107 L. Garbuio, B. Lewandowski, P. Wilhelm, L. Ziegler, M. Yulikov, H. Wennemers and G. Jeschke, Chem. - Eur. J., 2015, 21, 10747-10753.

108 S. Razzaghi, M. Qi, A. I. Nalepa, A. Godt, G. Jeschke, A. Savitsky and M. Yulikov, J. Phys. Chem. Lett., 2014, 5, 3970-3975.

109 G. Jeschke, V. Chechik, P. Ionita, A. Godt, H. Zimmermann, J. Banham, C. R. Timmel, D. Hilger and H. Jung, Appl. Magn. Reson., 2006, 30, 473-498.

110 C. Gemperle, G. Aebli, A. Schweiger and R. R. Ernst, J. Magn. Reson., 1990, 88, 241-256.

111 P. Hu and S. R. Hartmann, Phys. Rev. B: Solid State, 1974, 9, 1-13.

112 A. Savitsky, A. A. Dubinskii, M. Flores, W. Lubitz and K. Mobius, J. Phys. Chem. B, 2007, 111, 6245-6262.

113 G. Jeschke and Y. Polyhach, Phys. Chem. Chem. Phys., 2007, 9, 1895-1910. 
114 R. B. Zaripov, V. I. Dzhabarov, A. A. Knyazev, Y. G. Galyametdinov and L. V. Kulik, Appl. Magn. Reson., 2011, 40, 11-19.

115 A. A. Kuzhelev, O. A. Krumkacheva, G. Y. Shevelev, M. Yulikov, M. V. Fedin and E. G. Bagryanskaya, Phys. Chem. Chem. Phys., 2018, 20, 10224-10230.
116 A. M. Bowen, M. W. Jones, J. E. Lovett, T. G. Gaule, M. J. McPherson, J. R. Dilworth, C. R. Timmel and J. R. Harmer, Phys. Chem. Chem. Phys., 2016, 18, 5981-5994.

117 S. K. Misra, P. P. Borbat and J. H. Freed, Appl. Magn. Reson., 2009, 36, 237. 